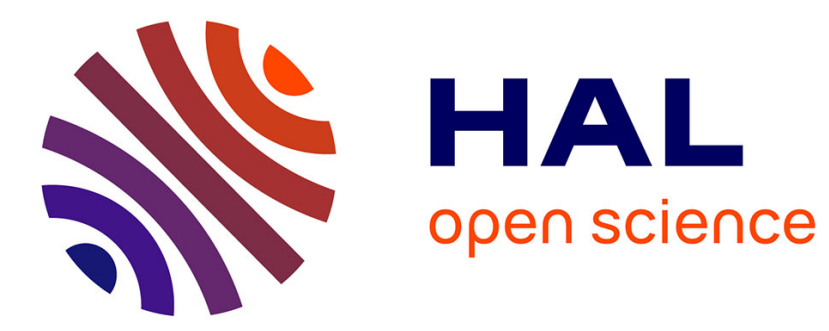

\title{
Periodic and quasi-periodic solutions for multi-instabilities involved in brake squeal
}

Nicolas Coudeyras, Samuel Nacivet, Jean-Jacques Sinou

\section{To cite this version:}

Nicolas Coudeyras, Samuel Nacivet, Jean-Jacques Sinou. Periodic and quasi-periodic solutions for multi-instabilities involved in brake squeal. Journal of Sound and Vibration, 2009, 328 (4-5), pp.520540. 10.1016/j.jsv.2009.08.017 . hal-00425156

\section{HAL Id: hal-00425156 https://hal.science/hal-00425156}

Submitted on 25 Sep 2012

HAL is a multi-disciplinary open access archive for the deposit and dissemination of scientific research documents, whether they are published or not. The documents may come from teaching and research institutions in France or abroad, or from public or private research centers.
L'archive ouverte pluridisciplinaire HAL, est destinée au dépôt et à la diffusion de documents scientifiques de niveau recherche, publiés ou non, émanant des établissements d'enseignement et de recherche français ou étrangers, des laboratoires publics ou privés. 


\title{
Periodic and quasi-periodic solutions for multi-instabilities involved in brake squeal
}

\author{
N. Coudeyras ${ }^{a, b}$, S. Nacivet ${ }^{b}$ and J-J. Sinou ${ }^{a}$ \\ ${ }^{a}$ Laboratoire de Tribologie et Dynamique des Systèmes UMR-CNRS 5513, Ecole Centrale de Lyon, 36 \\ avenue Guy de Collongue,69134 Ecully Cedex, France. \\ ${ }^{b}$ PSA Peugeot Citroën, 18 rue des Fauvelles, 92250 La Garenne Colombes, France.
}

Keywords : Brake squeal, multi-instabilities, stability analysis, nonlinear limit cycles.

\begin{abstract}
This paper is devoted to the computation of nonlinear dynamic steady-state solutions of autonomous systems subjected to multi-instabilities and proposes a new non-linear method for predicting periodic and quasi-periodic solutions intended for application to the disc brake squeal phenomenon. Firstly, finite element models of a pad and a disc are reduced to include only their contact nodes by using a Craig and Bampton strategy. Secondly, a complex eigenvalue analysis is performed showing two unstable modes for a wide range of friction coefficients, after which a Generalized Constrained Harmonic Balance Method (GCHBM) is presented. This method can compute nonlinear periodic or pseudo-periodic responses depending on the number of unstable frequencies. The numerical results are in good agreement with those of time marching methods.
\end{abstract}

\section{Introduction}

Disc brake squeal is still an issue for engineers and scientific communities. A great deal of work has been done in previous decades to understand the mechanism underlying squeal noise and formulate solutions for eradicating it. Kinkaid et al. [1] and Ouyang et al. [2] have performed extensive reviews of this phenomenon. The first models of disc brake squeal were built with one degree of freedom (dof) systems in which velocity friction dependency was considered as the squeal mechanism [3]. Then, Spurr [4] developed a sprag-slip model with a constant friction coefficient and highlighted squeal conditions. A generalization of this phenomenon was studied with geometrical coupling between bodies $[5,6]$. The mode coupling effect due to friction was considered and it was shown that squeal could occur even if the friction coefficient is constant in relation to sliding velocity. This mechanism is now commonly considered as the first cause of squeal generation and many works are based on the mode coupling effect for squeal analysis. The continued development of computer software has led to the use of the finite element method to study large and complex refined systems (see for example references $[7,8]$ ). The primary tool for predicting squeal propensity is complex eigenvalue analysis. Eigenvalues with positive real parts are related to unstable modes that are responsible for squeal generation, whereas negative real parts are related to stable modes. Stability and instability areas are then plotted versus system parameters and can provide clues for squeal-free brake design. Nevertheless, the literature states [9-11] that the computation of dynamic steady-states is increasingly employed because it leads to improved comprehension of the nonlinear aspects of the system and facilitates robust brake design. A major drawback of time marching methods is the CPU time consumed for computing steady-state responses. Besides, alternative methods 
in the frequency domain have been developed in order to enhance computation of stationary nonlinear dynamic solutions. Mention can be made of the direct Harmonic Balance Method (HBM), which is the most popular technique and used by many authors [6,12]. Rather than computing all the transient parts in the time domain, Harmonic Balance Methods are designed only to compute the Fourier coefficients of the steady-state solution by balancing terms between displacements and nonlinear forces. A derivation of Harmonic Balance Methods, called the Constrained Harmonic Balance Method (CHBM), developed in reference [13], is used for autonomous systems in which no periodic excitation exists.

In this paper, we perform a generalization of the Constrained Harmonic Balance Method (CHBM) by applying it to autonomous systems subjected to multiple unstable modes. In general, the ratio between two modal frequencies is not an integer and such frequencies are considered incommensurate. Consequently, such modes involved in the dynamic response produce pseudo-periodic solutions and HBM based-methods can be designed to deal with multiple frequencies. Since disc brake squeal is related to autonomous dynamic systems, several specific extensions are presented, leading to a proper algorithm based on the Harmonic Balance Method. This algorithm can compute the steady-state responses of autonomous systems with multiple input frequencies linked to unstable modes identified in the stability analysis. This paper is divided into four parts: firstly, a numerical model is presented with the modeling hypotheses. Secondly, a stability analysis is performed, highlighting two unstable modes for a given range of parameter. Then, a Generalized Constrained Harmonic Balance Method (GCHBM) designed for computing nonlinear steady-state responses is presented. The last part is devoted to the presentation and discussion of the results. A comparison with a time marching method is carried out with an evaluation of computational performances.

\section{Modeling of the Brake System}

The nonlinear system considered here is a reduced finite element model of a pad and disc and is based on a previous study by Sinou et al [14]. Based on a finite element procedure, each component of the system shown in Figure (1) is reduced by using a Craig and Bampton strategy [15]. Since highly nonlinear phenomena appear at the disc/pad interface, it is necessary to conserve physical dofs on both components at this location to ensure, for example, fine management of the contact status between the pad and the disc. Nine contact nodes are conserved and distributed equally on the frictional pad surface. Since the disc and pad meshes are compatible at the selected nodes, nine face to face nodes are kept on the frictional disc surface. Using only nine contact nodes may not provide an accurate description of the complex behavior of the surfaces, but it seems adequate for our purposes in terms of behavior and size for validating the new algorithm presented in the following. It should be noted that the nodes remain opposite each other during the computations and a relative velocity is imposed at the contact nodes to generate friction forces. Certain generalized dofs are included in the final reduced model, as keeping only static nodes gives a poor dynamic description of the super-elements. This procedure adds the first $n$ constrained modes in the reduction basis. We choose $n=50$ for both components corresponding to a cut-off frequency equal to $10 \mathrm{kHz}$ for both super-elements. Finally, an equation of motion is derived:

$$
\mathbf{M} \ddot{\mathbf{U}}+\mathbf{D U}+\mathbf{K U}+\mathbf{F}_{\mathrm{nl}}(\mathbf{U})=\mathbf{F}_{\text {out }}
$$

where $\mathbf{M}, \mathbf{D}, \mathbf{K}$ are the linear mass, damping and stiffness matrices respectively. $\mathbf{F}_{\mathrm{nl}}(\mathbf{U})$ is related to a vector of nonlinear forces occurring at the disc/pad interface and $\mathbf{F}_{\text {out }}$ is the vector of external forces. $\ddot{\mathbf{U}}, \dot{\mathbf{U}}, \mathbf{U}$ are respectively acceleration, velocity and displacement vectors. The model has 9 contact elements and 158 dofs including 54 nonlinear dofs and 104 linear dofs. Contacts are included in the physical 


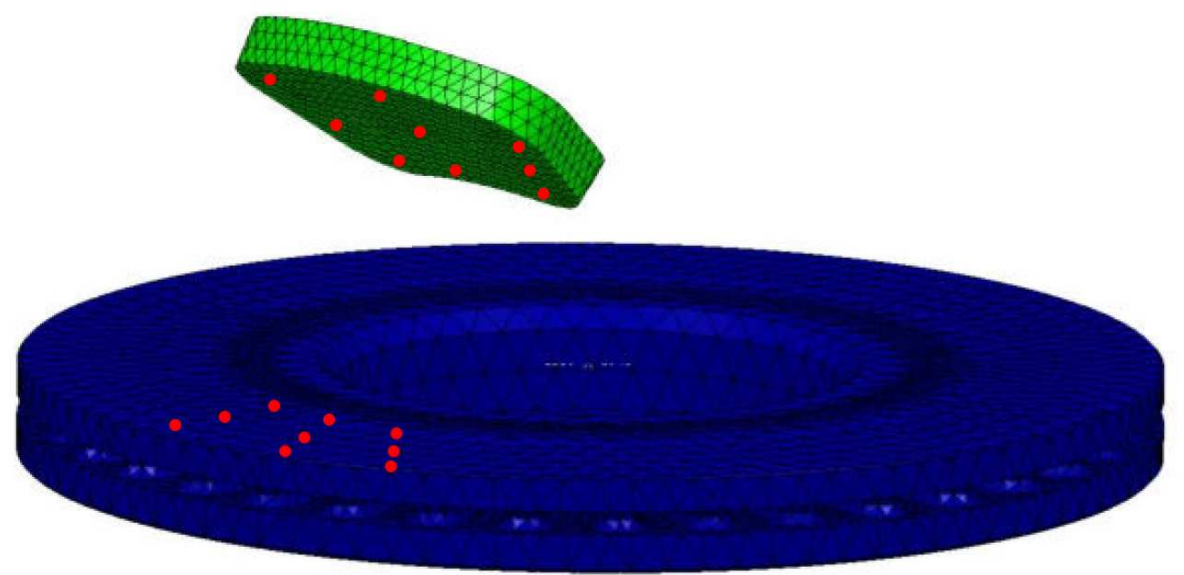

Figure 1: Split view of the finite element model of the brake system. The red dots represent the contact nodes

systems to add constraints in the equation of motion (1). For convenience, we choose to consider the penalty method. Springs are added at the disc/pad interface to impose contact conditions. Measurements of pad compressibility show a nonlinear relationship between the pressure and the displacement. This effect is included at the interfaces where nonlinear contact stiffnesses are considered in our model. Finally, the mathematical function used to describe the contact force is:

$$
F_{\text {contact }, \mathrm{i}}= \begin{cases}k_{1}\left(u_{1}-u_{2}\right)+k_{\mathrm{nl}}\left(u_{1}-u_{2}\right)^{3} & \text { if }\left(u_{1}-u_{2}\right)>0 \\ 0 & \text { otherwise }\end{cases}
$$

where $u_{1}$ and $u_{2}$ are respectively displacements of contact nodes of the pad and disc at contact element $i$. For the frictional definition, we consider a simplified Coulomb law with a constant friction coefficient without stick-slip motion. Moreover, a unidirectional friction effort is considered. Then a friction force $F_{\mathrm{f}, \mathrm{i}}$ located at node $i$ is derived from a contact effort $F_{\text {contact,i }}$ with:

$$
F_{\mathrm{f}, \mathrm{i}}=\mu F_{\text {contact,i }} \operatorname{sgn}\left(v_{\mathrm{r}, \mathrm{i}}\right)
$$

where $\mu$ is the friction coefficient and $v_{\mathrm{r}, \mathrm{i}}$ the relative velocity between the disc and pad at node $i$. The damping matrix $\mathbf{D}$ is built by considering a Rayleigh damping with $\alpha$ and $\beta$ chosen to obtain modal damping $\zeta=0.1$ on non-frictional coupled modes $900 \mathrm{~Hz}$ and $940 \mathrm{~Hz}$. The external force is directly applied on the back pad on four nodes for almost piston like pressure distribution. Table 1 provides the model parameters. To enhance understanding, it may be noted that the chosen finite element model (with the contact and damping assumption) does not attempt to capture all effects realistically. This modeling has been chosen to illustrate a suitable range of behavior and to investigate the efficiency of the proposed non-linear method.

\section{Static computation and stability analysis}

The classical tool for predicting unstable modes in squeal analysis is a linear computation consisting in finding unstable modes around a linearized static position. The first step considers only the static part of 


\begin{tabular}{|c|c|}
\hline \multicolumn{2}{|c|}{ Pad } \\
\hline Young Modulus $E(G P a)$ & 2 \\
\hline Poison ration $\nu$ & 0.1 \\
\hline Density $\rho \quad\left(k g . m^{-3}\right)$ & 2500 \\
\hline \multicolumn{2}{|c|}{ Disc } \\
\hline Young Modulus $E(G P a)$ & 125 \\
\hline Poison ration $\nu$ & 0.3 \\
\hline Density $\rho \quad\left(k g . m^{-3}\right)$ & 7200 \\
\hline
\end{tabular}

Table 1: Physical characteristics

\begin{tabular}{|cl|c|}
\hline$\alpha$ & $(s)$ & $1.6 e^{-7}$ \\
\hline$\beta$ & $\left(s^{-1}\right)$ & 6.1 \\
\hline$k_{l}$ & $\left(N \cdot m^{-1}\right)$ & $1.8 e^{5}$ \\
\hline$k_{n l}$ & $\left(N . m^{-3}\right)$ & $5 e^{9}$ \\
\hline$F_{\text {out }}$ & $(N)$ & 7000 \\
\hline
\end{tabular}

Table 2: Parameter values

the system with nonlinear terms at the contact interface:

$$
\mathbf{K U}_{0}+\mathbf{F}_{\mathrm{nl}}\left(\mathbf{U}_{0}\right)=\mathbf{F}_{\text {out }}
$$

Then, a nonlinear steady sliding equilibrium position $\mathbf{U}_{0}$ is computed and its stability is analyzed by using a perturbation step procedure:

$$
\mathbf{U}=\mathbf{U}_{0}+\overline{\mathbf{U}}
$$

substituting the previous solution in the equation of motion (1) with a linearization step gives:

$$
\mathbf{M} \ddot{\bar{U}}+\mathbf{D} \dot{\bar{U}}+\left(\mathbf{K}+\mathbf{J}_{\mathbf{n l}}\right) \overline{\mathbf{U}}=\mathbf{0}
$$

where $\mathbf{J}_{\mathbf{n l}}$ is the frictional contact Jacobian matrix derived from linearized expressions of contact efforts. An elementary Jacobian matrix $\mathbf{J}_{\mathrm{nl}_{\mathbf{i}}}$ at contact node $i$ takes the form:

$$
\mathbf{J}_{\mathbf{n l}_{\mathbf{i}}}=\left[\begin{array}{ll}
\left.\frac{\partial F_{n l_{1, i}}}{\partial u_{1, i}}\right|_{u_{0}} & \left.\frac{\partial F_{n l_{1, i}}}{\partial u_{2, i}}\right|_{u_{0}} \\
\left.\frac{\partial F_{n l_{2, i}}}{\partial u_{1, i}}\right|_{u_{0}} & \left.\frac{\partial F_{n l_{2, i}}}{\partial u_{2, i}}\right|_{u_{0}}
\end{array}\right]
$$

Note that $\mathbf{J}_{\mathbf{n l}}$ is asymmetric since the model contains friction forces. The complex eigenvalue computation of (6) then gives the local stability of the nonlinear system. Eigenvalues take the form

$$
\lambda_{j}=a_{j}+i \omega_{j}
$$

where $\omega_{j}$ is the natural pulsation in rad.s $s^{-1}$ of mode $j$, defined as $\omega_{j}=2 \pi f_{j}$ with $f_{j}$ being the natural frequency in $\mathrm{Hz}$ and $a_{j}$ its associated real part. The modal damping can be expressed as $\zeta_{j}=$ $-2 a_{j} / \omega_{j}$, since as long as the real part remains negative the associated mode is stable with a positive modal damping $\zeta_{j}>0$. However, when $a_{j}$ becomes positive, the associated mode has a negative modal 
a
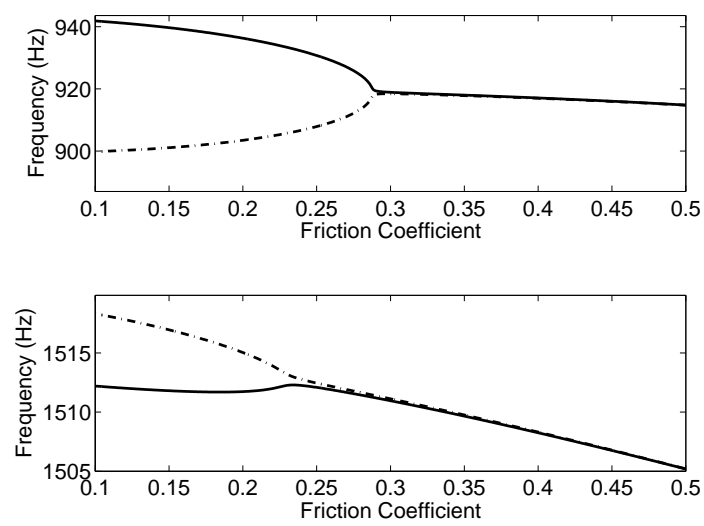

b
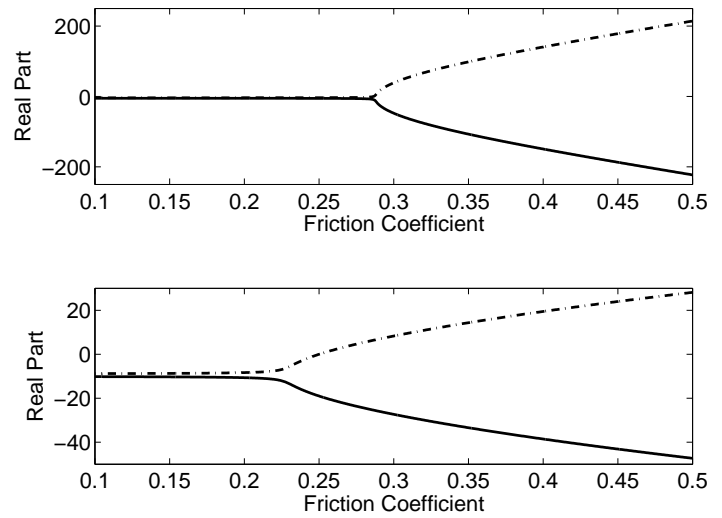

Figure 2: Evolution of the Frequencies (a) and Real Parts (b) with the friction coefficient (dashed lines: unstable modes)

damping $\zeta_{j}<0$ that supplies energy. Then, mode $j$ is considered as unstable and can generate vibrations at its natural frequency, resulting in squeal noise emission. Figure 2 shows the evolution of (a) the frequencies and (b) the real parts. We clearly see the frequency coalescence of non-frictional coupled modes due to friction. An unstable mode appears at the Hopf bifurcation point where $\mu=0.26$ and the frequency is $f_{2}=1514 \mathrm{~Hz}$. The real parts split with one becoming positive while the other remains in the negative area. Frequency coalescence is not perfect due to unevenly distributed damping between modes [16]. Another unstable mode at $f_{1}=920 \mathrm{~Hz}$ appears for a larger friction coefficient value $(\mu=0.28)$. Frequency coalescence is perfect because both non-frictional coupled modes are equally damped, as seen previously. Both modes are considered as unstable above this second Hopf bifurcation point. In brief, we distinguish three main areas in the coalescence pattern of Figure (2). The first considers a friction coefficient under 0.26 where no instability occurs. The second is between the two Hopf bifurcation points (i.e. $\mu$ ranges from 0.26 to 0.28 ) where only mode $f_{2}$ at $1514 \mathrm{~Hz}$ is unstable. The third part starts from $\mu=0.28$ until $\mu=1$, where two modes are unstable at two distinct frequencies $f_{1}$ and $f_{2}$. Note that the growth rate of the real part of the first mode $f_{1}$ is the highest in the model.

This simple model raises one of the problems of the squeal phenomenon: that of the vibrations when more than one mode is considered as unstable. To solve this problem, and since the real parts of the unstable modes are not an indicator of dynamic behavior, temporal analysis is generally employed for the whole system (1). Hence, we are able to obtain a good representation of nonlinear behavior, providing a great deal of information such as vibration level and frequency components. However, the major drawback of this approach is the computation time required to obtain the dynamic steady-state of the nonlinear autonomous system. An alternative method known as CHBM [13] can be used to compute the dynamic steady-state of autonomous systems with one unstable mode faster than with time marching methods. An extension of CHBM for dealing with a wide range of autonomous systems with multiple instabilities is proposed in the following. 


\section{Generalized Harmonic Balance Method}

\subsection{Quasi periodic functions}

Responses are no longer periodic when oscillatory systems are subjected to $p$ incommensurable frequencies. The nonlinear oscillations contain the frequency components of any linear combination of the incommensurable frequency components

$$
k_{1} \omega_{1}+k_{2} \omega_{2}+\cdots+k_{j} \omega_{j}+\cdots+k_{p} \omega_{p}
$$

with $k_{i}=-N_{h},-N_{h}+1, \ldots,-1,0,1, \ldots, N_{h}-1, N_{h}$ for $i=1, \ldots, p . N_{h}$ defines the order for each fundamental frequency and $p$ the number of incommensurable frequencies.

Thus the dynamic solution of equation (1) can be expressed with a generalized Fourier series such that:

$$
\mathbf{U}(t) \approx \sum_{k_{1}=-N_{h}}^{N_{h}} \ldots \sum_{k_{p}=-N_{h}}^{N_{h}} \mathbf{a}_{\mathbf{k}_{1} \ldots \mathbf{k}_{\mathbf{p}}} \cos \left(k_{1} \omega_{1}+\ldots+k_{p} \omega_{p}\right) t+\mathbf{b}_{\mathbf{k}_{\mathbf{1}} \ldots \mathbf{k}_{\mathbf{p}}} \sin \left(k_{1} \omega_{1}+\ldots+k_{p} \omega_{p}\right) t
$$

$\mathbf{a}_{\mathbf{k}_{1}, \ldots, \mathbf{k}_{\mathbf{p}}}$ and $\mathbf{b}_{\mathbf{k}_{1}, \ldots, \mathbf{k}_{\mathbf{p}}}$ define the unknown Fourier coefficients of any linear combinations of the incommensurable frequency components $\omega_{1}, \omega_{2}, \ldots, \omega_{p}$ that have been defined previously in (9).

The previous expression can be rewritten in a condensed form

$$
\mathbf{U}(t)=\mathbf{a}_{0}+\sum_{\mathbf{k} \in \mathbb{Z} \mathbf{p}} \mathbf{a}_{\mathbf{k}} \cos (\mathbf{k} \cdot \boldsymbol{\omega}) t+\sum_{\mathbf{k} \in \mathbb{Z} \mathbf{p}} \mathbf{b}_{\mathbf{k}} \sin (\mathbf{k} \cdot \boldsymbol{\omega}) t
$$

where the (.) denotes the dot product, $\mathbf{k}$ is the harmonic number vector of each frequency direction and $\boldsymbol{\omega}$ is the vector of the $p$ incommensurable frequencies considered in the solution. For convenience, it is wise to deal with a multiple time parameter $\tau$ such that

$$
\tau=\omega t
$$

and equation (11) is rewritten as

$$
\mathbf{U}(\boldsymbol{\tau})=\mathbf{a}_{0}+\sum_{\mathbf{k} \in \mathbb{Z}^{\mathbf{p}}} \mathbf{a}_{\mathbf{k}} \cos (\mathbf{k} \cdot \boldsymbol{\tau})+\sum_{\mathbf{k} \in \mathbb{Z}^{\mathbf{p}}} \mathbf{b}_{\mathbf{k}} \sin (\mathbf{k} \cdot \boldsymbol{\tau})
$$

where $\tau=\left[\tau_{1} \ldots \tau_{p}\right]$ is a non dimensional time parameter and refers to the hyper-time concept proposed by [17]. Consequently, rather than dealing with a single time domain $t \in \mathbb{R}_{+}$for solution $\mathbf{U}(t)$, a multiple periodic time domain $\tau \in \mathbb{R}_{+}^{p}$ is considered where each dimension $\tau_{j}$ corresponds to one incommensurable fundamental frequency identified in the solution. Therefore (13) is $2 \pi$ periodic on every hyper-time dimension of $\boldsymbol{\tau} . \mathbf{U}(\boldsymbol{\tau})$ represents $\mathbf{U}(t)$ in a $p$ dimensional time space where each frequency is independent from the others. For example, Schilder et al. [18] define torus functions which represent the hyper-time domain in a 3 dimensional space for 2 incommensurate frequencies. An analogy with numerical image processing can be considered to illustrate the hyper-time concept when applying a filter on an image. It can be considered as a visual signal depending on both parameters, its two orthogonal directions $(x, y)$ that are similar to $\tau_{1}$ and $\tau_{2}$ in a hyper-time domain.

Theoretically, (13) is able to treat a great range of aperiodic dynamic systems where a finite number of $p$ incommensurable frequencies have been identified. A definition given by [17] for defining $N_{h}$ harmonics in a multiple Fourier series is: 


$$
\sum_{j=1}^{p}\left|k_{j}\right| \leq N_{h}
$$

This choice is justified by the fact that the major part of the signal energy is generally included in the very first harmonics and the very first coupling frequencies.

Equation (14) will be used in the following for the multiple Fourier series truncation.

\subsection{Generalized Harmonic Balance Method}

Substituting (11) in the behavior equation (1) and considering equation (14) gives:

$$
\begin{aligned}
\mathbf{R}(t) \approx & \sum_{\mathbf{k} \in \mathbb{Z}_{N_{h}}^{n}}\left[\left(\mathbf{K}-(\mathbf{k} \cdot \boldsymbol{\omega})^{2} \mathbf{M}\right) \mathbf{a}_{k}+((\mathbf{k} \cdot \boldsymbol{\omega}) \mathbf{D}) \mathbf{b}_{k}\right] \cos (\mathbf{k} \cdot \boldsymbol{\tau})+ \\
& \sum_{\mathbf{k} \in \mathbb{Z}_{N_{h}}^{n}}\left[\left(\mathbf{K}-(\mathbf{k} \cdot \boldsymbol{\omega})^{2} \mathbf{M}\right) \mathbf{b}_{k}-((\mathbf{k} \cdot \boldsymbol{\omega}) \mathbf{D}) \mathbf{a}_{k}\right] \sin (\mathbf{k} \cdot \boldsymbol{\tau})+\mathbf{F}_{\mathrm{nl}}\left(\mathbf{a}_{k}, \mathbf{b}_{k}\right)-\mathbf{F}_{\text {out }}
\end{aligned}
$$

Since sine and cosine are orthogonal functions, they are used as bases and we use a Galerkin procedure for computing Fourier coefficients:

$$
\begin{gathered}
\int_{0}^{2 \pi} \ldots \int_{0}^{2 \pi} \mathbf{R} \cos \left(k_{1} \cdot \tau_{1}+\ldots+k_{p} \cdot \tau_{p}\right) d \tau_{1} \ldots d \tau_{p}=0 \text { for all } k_{j} \text { such } \sum_{j=1}^{p}\left|k_{j}\right| \leq N_{h} \\
\int_{0}^{2 \pi} \ldots \int_{0}^{2 \pi} \mathbf{R} \sin \left(k_{1} \cdot \tau_{1}+\ldots+k_{p} \cdot \tau_{p}\right) d \tau_{1} \ldots d \tau_{p}=0 \text { for all } k_{j} \text { such } \sum_{j=1}^{p}\left|k_{j}\right| \leq N_{h}
\end{gathered}
$$

And the following set of algebraic equations is derived:

$$
\Lambda \tilde{\mathbf{z}}+\tilde{\mathbf{F}}_{\mathrm{nl}}(\tilde{\mathbf{z}})=\tilde{\mathbf{F}}_{\text {out }}
$$

Where $\Lambda$ refers to the block diagonal dynamic stiffness matrix:

$$
\boldsymbol{\Lambda}=\left[\begin{array}{cccccc}
\mathbf{K} & \mathbf{0} & \mathbf{0} & \mathbf{0} & \mathbf{0} & \mathbf{0} \\
\mathbf{0} & \boldsymbol{\Lambda}_{1} & \mathbf{0} & \mathbf{0} & \mathbf{0} & \mathbf{0} \\
\mathbf{0} & \mathbf{0} & \ddots & \mathbf{0} & \mathbf{0} & \mathbf{0} \\
\mathbf{0} & \mathbf{0} & \mathbf{0} & \boldsymbol{\Lambda}_{i} & \mathbf{0} & \mathbf{0} \\
\mathbf{0} & \mathbf{0} & \mathbf{0} & \mathbf{0} & \ddots & \mathbf{0} \\
\mathbf{0} & \mathbf{0} & \mathbf{0} & \mathbf{0} & \mathbf{0} & \boldsymbol{\Lambda}_{N c}
\end{array}\right]
$$

with

$$
\boldsymbol{\Lambda}_{i}=\left[\begin{array}{cc}
-(\mathbf{k} \cdot \boldsymbol{\omega})^{2} \mathbf{M}+\mathbf{K} & (\mathbf{k} \cdot \boldsymbol{\omega}) \mathbf{D} \\
-(\mathbf{k} \cdot \boldsymbol{\omega}) \mathbf{D} & -(\mathbf{k} \cdot \boldsymbol{\omega})^{2} \mathbf{M}+\mathbf{K}
\end{array}\right] \text { for } i \in\left[1, N_{c}\right]
$$

$N_{c}$ represents the total number of frequency components including all harmonic terms up to $N_{h}$ of each frequency direction and all the coupling frequencies chosen by using (14). They must also be 
positive. Therefore $N_{c}$ depends on $\omega_{k}$ values. A particular case where $p=2$ is studied in the following and thus $N_{c}$ is defined.

$\tilde{\mathbf{z}}, \tilde{\mathbf{F}}_{\mathrm{nl}}(\tilde{\mathbf{z}})$ and $\tilde{\mathbf{F}}_{\text {out }}$ are respectively the Fourier coefficient vectors of displacement, nonlinear frictional contact forces and external forces of the system. They are expressed as:

$$
\begin{gathered}
\tilde{\mathbf{z}}=\left[\begin{array}{llllll}
\mathbf{a}_{0} & \mathbf{a}_{1} & \mathbf{b}_{1} & \cdots & \mathbf{a}_{\mathrm{N}_{\mathrm{c}}} & \mathbf{b}_{\mathrm{N}_{\mathrm{c}}}
\end{array}\right] \\
\tilde{\mathbf{F}}_{\mathrm{nl}}=\left[\begin{array}{llllll}
\tilde{\mathbf{F}}_{\mathrm{nl}, 0} & \tilde{\mathbf{F}}_{\mathrm{nl}, 1}^{\mathrm{a}} & \tilde{\mathbf{F}}_{\mathrm{nl}, 1}^{\mathbf{b}} & \cdots & \tilde{\mathbf{F}}_{\mathrm{nl}, \mathrm{N}_{\mathrm{c}}}^{\mathbf{a}} & \tilde{\mathbf{F}}_{\mathrm{nl}, \mathrm{N}_{\mathrm{c}}}^{\mathbf{b}}
\end{array}\right] \\
\tilde{\mathbf{F}}_{\text {out }}=\left[\begin{array}{llllll}
\tilde{\mathbf{F}}_{\text {out }, 0} & \tilde{\mathbf{F}}_{\text {out }, 1}^{\mathbf{a}} & \tilde{\mathbf{F}}_{\text {out }, 1}^{\mathrm{b}} & \cdots & \tilde{\mathbf{F}}_{\text {out, } \mathrm{N}_{\mathrm{c}}}^{\mathbf{a}} & \tilde{\mathbf{F}}_{\text {out }, \mathrm{N}_{\mathrm{c}}}^{\mathbf{b}}
\end{array}\right]
\end{gathered}
$$

Since the behavior of $\tilde{\mathbf{F}}_{\mathrm{nl}}$ is nonlinear with respect to the displacement vector, equation (17) must be solved iteratively by using Newton-Raphson root-finding algorithms. The analytical computation of $\tilde{\mathbf{F}}_{\mathrm{nl}}$ in the frequency domain is prohibitive when defined as a piecewise nonlinear function of displacement. To get round this issue, certain techniques have been developed for computing nonlinear terms. The Incremental Harmonic Balance Method (IHBM) or High Dimensional Harmonic Balance Method (HDHBM) $[19,20]$ is applied to nonlinear systems under multiple excitation frequencies. The nonlinear treatment of Fourier coefficients is performed by using multiple time scales where transformation matrices of equally spaced sub-time levels are built to compute the nonlinear Fourier coefficients. Cameron [21] proposes the Alternating-Frequency Time (AFT) method to compute the periodic nonlinear forces in the time domain and then extract their exact Fourier coefficients $\tilde{\mathbf{F}}_{\mathrm{nl}}$. As we use a hyper-time domain where the functions are $2 \pi$ periodic on every orthogonal time dimension, the generalization of the AFT can be extended to a $p$-dimensional frequency domain by using a $p$-dimensional FFT. The procedure is as follows:

$$
\tilde{\mathbf{z}} \stackrel{\text { IFFT }^{\mathbf{P}}}{\longrightarrow} \mathbf{U}(\boldsymbol{\tau}) \longrightarrow \mathbf{F}_{\mathrm{nl}}(\mathbf{U}(\boldsymbol{\tau})) \stackrel{\text { FFT }^{\mathbf{p}}}{\longrightarrow} \tilde{\mathbf{F}}_{\mathrm{nl}}(\tilde{\mathbf{z}})
$$

Once $\mathbf{F}_{\mathrm{nl}}(\mathbf{U}(\boldsymbol{\tau}))$ is evaluated, its Fourier coefficients are computed and injected into equation (17). In brief, GHBM is written as an objective function $J_{1}$ of a dynamic system subjected to multiple frequency inputs:

$$
J_{1}(\tilde{\mathbf{z}})=\left\|\boldsymbol{\Lambda} \tilde{\mathbf{z}}+\tilde{\mathbf{F}}_{\mathrm{nl}}(\tilde{\mathbf{z}})-\tilde{\mathbf{F}}_{\text {out }}\right\|<\epsilon_{1}
$$

where $\|$.$\| defines the euclidean norm and \epsilon_{1}$ is a chosen tolerance.

\subsection{Additional constraint equations}

This derivation of GHBM can be applied to a wide variety of dynamic systems exhibiting pseudoperiodic responses due to a finite number of identified exciting frequencies. As seen previously, disc brake squeal is equivalent to an autonomous system, i.e. the dynamic response implicitly depends on time or, in other words, no external excitation forces excite the structure. Thus GHBM gives the trivial solution in which the Fourier coefficients would be null except for the static components, even though a local minimum exists for the dynamic steady-state in the optimization domain [13]. The existence of both solutions is related to the nature of the dynamic system illustrated by equation (1). Under unstable static conditions, the system may or may not oscillate, depending only on the initial conditions or a perturbation of the system's parameters. Hence a set of additional equations has to be included in equation (17) to reach the minimum related to the dynamic solution. 
If we consider that the nonlinear response of an autonomous system is driven by $p$ unstable modes:

$$
\mathbf{U}(t)=\sum_{j=1}^{p} \boldsymbol{\Psi}_{\boldsymbol{j}} e^{\varphi_{j} t}
$$

where $\boldsymbol{\Psi}_{j}$ is an unstable mode and $\varphi_{j}=a_{j}+i \omega_{j}$ its eigenvalue. It can be seen that $\boldsymbol{\Psi}_{j}$ and $\varphi_{j}$ depend implicitly on $\mathbf{U}(t)$ since they are subjected to nonlinear effects thus a change in contact status. Hence, $\left[\Psi_{1} \ldots \Psi_{p}\right]^{\mathbf{T}}$ should be considered as unstable modes of a dynamic state with their corresponding complex eigenvalues $\left[\varphi_{1} \ldots \varphi_{p}\right]^{\mathbf{T}}$, as opposed to unstable modes of the sliding steady-state solution seen in section 3. Hence, when looking at equation (25), a null real part of $\varphi_{j}$ indicates that the dynamic response $\mathbf{U}_{j}(t)=\Psi_{j} e^{i \omega_{j} t}$ is stationary through time and oscillates with pulsation $\omega_{j}$. Therefore, when all $p$ unstable modes have a null real part, $\mathbf{U}_{j}(t)$ becomes a pseudo-periodic motion that is the steadystate response of the autonomous system considered. Therefore minimizing the real parts of eigenvalues $\varphi_{j}$ (with $j \in[1, p]$ ) in the optimization process would lead to the correct computation of the Fourier coefficients linked to the steady-state solution.

The computation of $\varphi_{j}$ is performed by considering an equivalent linear system to equation (1) and refers to the equivalent linear concept proposed by Iwan $[22,23]$ where the nonlinear terms $\mathbf{F}_{\mathbf{n l}}(\mathbf{U}(t))$ are replaced with a linear approximation matrix $\mathbf{J}_{\mathbf{n l}}$ such that:

$$
\zeta=\mathbf{F}_{\mathbf{n l}}(\mathbf{U}(t))-\mathbf{J}_{\mathbf{n l}} \mathbf{U}(t) \text { with } \zeta \rightarrow 0
$$

$\mathbf{J}_{\mathbf{n l}}$ refers to a time independent Jacobian matrix of the nonlinear temporal forces $\mathbf{F}_{\mathbf{n l}}(\mathbf{U}(t))$ at the dynamic stationary state $\mathbf{U}(t)$.

Since a Fourier Transformation is a linear application, $\mathbf{J}_{\mathbf{n l}}$ can also be computed in the frequency domain with Fourier coefficients:

$$
\zeta=\tilde{\mathbf{F}}_{\mathbf{n l}}(\tilde{\mathbf{z}})-\mathbf{J}_{\mathbf{n l}} \tilde{\mathbf{z}} \text { with } \zeta \rightarrow 0
$$

A good linear approximation of (1) is obtained when $\zeta \rightarrow 0$. Then (1) is substituted by:

$$
\mathbf{M} \ddot{\mathbf{U}}+\mathbf{D U}+\left(\mathbf{K}+\mathbf{J}_{\mathbf{n l}}\right) \mathbf{U}=\mathbf{F}_{\text {out }}
$$

By using a perturbation method, the complex eigenvalues $\varphi$ of equation (28) are computed and the $p$ real parts linked to the unstable modes are extracted and gathered in a vector, forming a second objective function $J_{2}$.

Secondly, since there is no external excitation, the $p$ nonlinear pulsations $\omega_{j}$ are undefined and thus have to be considered as $p$ independent unknowns. In brief, a Generalized Constrained Harmonic Balance Method (GCHBM) applicable to autonomous systems subjected to $p$ incommensurate frequency components is arranged in a set of two objective functions:

$$
\left\{\begin{array}{l}
J_{1}(\tilde{\mathbf{z}}, \boldsymbol{\Omega})=\left\|\boldsymbol{\Lambda}(\tilde{\mathbf{z}}, \boldsymbol{\Omega}) \tilde{\mathbf{z}}+\tilde{\mathbf{F}}_{\mathrm{nl}}(\tilde{\mathbf{z}}, \boldsymbol{\Omega})-\tilde{\mathbf{F}}_{\text {out }}\right\|<\epsilon_{1} \\
J_{2}(\tilde{\mathbf{z}}, \boldsymbol{\Omega})=\left\|\operatorname{Re}\left(\varphi_{\mathrm{j}}(\tilde{\mathbf{z}}, \boldsymbol{\Omega})\right)\right\|<\epsilon_{2} \text { with } j \in[1, p]
\end{array}\right.
$$

In more detail $J_{2}$ takes the form

$$
J_{2}(\tilde{\mathbf{z}}, \boldsymbol{\Omega})=\left\{\begin{array}{c}
\left\|\operatorname{Re}\left(\varphi_{1}(\tilde{\mathbf{z}}, \boldsymbol{\Omega})\right)\right\| \\
\left\|\operatorname{Re}\left(\varphi_{2}(\tilde{\mathbf{z}}, \boldsymbol{\Omega})\right)\right\| \\
\vdots \\
\left\|\operatorname{Re}\left(\varphi_{\mathrm{p}}(\tilde{\mathbf{z}}, \boldsymbol{\Omega})\right)\right\|
\end{array}\right\}
$$


with vector $\Omega$ of $p$ unknown frequencies:

$$
\boldsymbol{\Omega}=\left\{\begin{array}{c}
\omega_{1} \\
\omega_{2} \\
\vdots \\
\omega_{p}
\end{array}\right\}
$$

Computation is performed when $\left\langle J_{1}, J_{2}>\right.$ are respectively below chosen tolerances $\left\langle\epsilon_{1}, \epsilon_{2}\right\rangle$. Such a set of objective functions has $\left(n\left(1+2 N_{c}\right)+p\right)$ equations to be solved with $\left(n\left(1+2 N_{c}\right)+p\right)$ unknowns and is therefore well-determined. $n$ is the dimension of the dynamic system, $N_{c}$ is the total number of frequency combinations and $p$ is the number of unstable modes used in the solution.

\subsection{Reduction step}

The computation time of any nonlinear system is related to the number of unknowns so any reduction in the size of the system would increase performance. The authors of [24] propose a reduction method for nonlinear systems studied in the frequency domain without loss of accuracy. After reorganizing linear and nonlinear dofs $\tilde{\mathbf{z}}_{\mathrm{nl}}$ and $\tilde{\mathbf{z}}_{\mathrm{ln}}$, the system described by equation (17) can be expressed as follows:

$$
\left[\begin{array}{cc}
\boldsymbol{\Lambda}_{\mathrm{ln}, \mathrm{ln}} & \boldsymbol{\Lambda}_{\mathrm{ln}, \mathrm{nl}} \\
\boldsymbol{\Lambda}_{\mathrm{nl}, \mathrm{ln}} & \boldsymbol{\Lambda}_{\mathrm{nl}, \mathrm{nl}}
\end{array}\right]\left\{\begin{array}{c}
\tilde{\mathbf{z}}_{\mathrm{ln}} \\
\tilde{\mathbf{z}}_{\mathrm{nl}}
\end{array}\right\}+\left\{\begin{array}{c}
\mathbf{0} \\
\tilde{\mathbf{F}}_{\mathrm{nl}}
\end{array}\right\}=\left\{\begin{array}{c}
\tilde{\mathbf{F}}_{\text {out }, \mathrm{ln}} \\
\tilde{\mathbf{F}}_{\text {out }, \mathrm{nl}}
\end{array}\right\}
$$

and equation (17) is rewritten in term of nonlinear components such that:

$$
\boldsymbol{\Lambda}_{\mathrm{eq}} \tilde{\mathbf{z}}_{\mathrm{nl}}+\tilde{\mathbf{F}}_{\mathrm{nl}}\left(\tilde{\mathbf{z}}_{\mathrm{nl}}\right)=\tilde{\mathbf{F}}_{\mathrm{eq}}
$$

with

$$
\boldsymbol{\Lambda}_{\mathrm{eq}}=\boldsymbol{\Lambda}_{\mathrm{nl}, \mathrm{nl}}-\boldsymbol{\Lambda}_{\mathrm{nl}, \mathrm{ln}}\left(\boldsymbol{\Lambda}_{\mathrm{ln}, \mathrm{ln}}\right)^{-\mathbf{1}} \boldsymbol{\Lambda}_{\mathrm{ln}, \mathrm{nl}}
$$

and

$$
\tilde{\mathbf{F}}_{\text {eq }}=\tilde{\mathbf{F}}_{\text {out }, \mathrm{nl}}-\boldsymbol{\Lambda}_{\mathrm{nl}, \ln }\left(\boldsymbol{\Lambda}_{\ln , \ln }\right)^{-1} \tilde{\mathbf{F}}_{\text {out }, \ln }
$$

This step reduces the number of equations from $\left(n\left(2 N_{c}+1\right)+p\right)$ to $\left(n_{n l}\left(2 N_{c}+1\right)+p\right)$ where $n$ and $n_{n l}$ are the numbers of total dofs and nonlinear dofs respectively. Thus this reduction step is very efficient for large systems with only few nonlinear dofs. When $\tilde{\mathbf{z}}_{\mathrm{nl}}$ is known, $\tilde{\mathbf{z}}_{\mathrm{ln}}$ is easily obtained by:

$$
\tilde{\mathbf{z}}_{\ln }=\boldsymbol{\Lambda}_{\ln , \ln }^{-1}\left(\tilde{\mathbf{F}}_{\text {out }, \ln }-\boldsymbol{\Lambda}_{\ln , \mathrm{nl}} \tilde{\mathbf{z}}_{\mathrm{nl}}\right)
$$

Hence, a reduced form of equation (29) is:

$$
\left\{\begin{array}{l}
J_{1}\left(\tilde{\mathbf{z}}_{\mathrm{nl}}, \boldsymbol{\Omega}\right)=\left\|\boldsymbol{\Lambda}_{\mathrm{eq}}\left(\tilde{\mathbf{z}}_{\mathrm{nl}}, \boldsymbol{\Omega}\right) \tilde{\mathbf{z}}_{\mathrm{nl}}+\tilde{\mathbf{F}}_{\mathrm{nl}}\left(\tilde{\mathbf{z}}_{\mathrm{nl}}, \boldsymbol{\Omega}\right)-\tilde{\mathbf{F}}_{\mathrm{eq}}\right\|<\epsilon_{1} \\
J_{2}\left(\tilde{\mathbf{z}}_{\mathrm{nl}}, \boldsymbol{\Omega}\right)=\left\|\operatorname{Re}\left(\varphi_{\mathrm{j}}\left(\tilde{\mathbf{z}}_{\mathrm{nl}}, \boldsymbol{\Omega}\right)\right)\right\|<\epsilon_{2} \text { with } j \in[1, p]
\end{array}\right.
$$

Computation is done when $\left\langle J_{1}, J_{2}\right\rangle$ are respectively below chosen tolerances $\left\langle\epsilon_{1}, \epsilon_{2}\right\rangle$. Figure 3 represents the general algorithm procedure of a GCHBM. 


\subsection{GCHBM initialization}

Regarding time marching methods, optimization processes need a starting point to find a solution in the optimization domain. A bad choice of initial conditions is synonymous with slow convergence. As explained in [13] and inspired by the concept of Complex Non Linear Modal Analysis (CNLMA) developed by Sinou et al. [25], Fourier coefficients of the first harmonic are initialized by the unstable mode vectors found in the stability analysis. This is based on the assumption that dynamic behavior is mainly driven by unstable modes. Thus for every first harmonic (in Fourier basis) of every unstable mode, the initial prediction takes the form:

$$
\tilde{\mathbf{z}}_{1, \mathrm{j}}=\eta\left(\mathbf{\Psi}_{\mathbf{j}}+\overline{\mathbf{\Psi}}_{\mathbf{j}}\right) \text { with } j \in[1, p]
$$

where $\boldsymbol{\Psi}_{\mathbf{j}}$ defines the $j^{\text {th }}$ nonlinear unstable mode shape, $\overline{\mathbf{\Psi}}_{\mathbf{j}}$ is its conjugate and $\eta$ is an arbitrarily chosen coefficient with a range from 20 to 60 to ensure convergence of the optimization problem. As can be observed, the pulsations are a priori unknown while a good initial estimate considers those found in the stability analysis.

\section{Application to squeal vibration}

\subsection{Brake model with two unstable modes}

As indicated previously in section 3, two modes are found to be unstable, with the potential to vibrate. Thus, $J_{2}$ and $\Omega$ are two-dimensional and take the following form:

$$
\begin{gathered}
J_{2}\left(\tilde{\mathbf{z}}_{\mathrm{nl}}, \boldsymbol{\Omega}_{\mathbf{1 - 2}}\right)=\left\{\begin{array}{l}
\left\|\operatorname{Re}\left(\varphi_{1}\left(\tilde{\mathbf{z}}_{\mathrm{nl}}, \boldsymbol{\Omega}_{\mathbf{1}-\mathbf{2}}\right)\right)\right\| \\
\left\|\operatorname{Re}\left(\varphi_{2}\left(\tilde{\mathbf{z}}_{\mathrm{nl}}, \boldsymbol{\Omega}_{\mathbf{1}-\mathbf{2}}\right)\right)\right\|
\end{array}\right\} \\
\boldsymbol{\Omega}_{\mathbf{1 - 2}}=\left\{\begin{array}{c}
\omega_{1} \\
\omega_{2}
\end{array}\right\}
\end{gathered}
$$

A geometric interpretation of $\mathbf{k} \in \mathbb{Z}^{2}$ (i.e. $p=2$ and $N_{h}=3$ ) is given in Figure 4 . It can be seen that the harmonic combinations are constrained by equation (14) and that the resulting frequencies are positive.

According to Figure 4, $N_{c}=12$ and as seen in section 2, the nonlinear terms are equal to $n_{n l}=54$. Thus the whole equation set has 1352 unknowns and equations. It should be noted that for the case where $k_{1}=k_{2}=0$ stands for the static Fourier coefficients and is not included in $N_{c}$.

\subsection{Nonlinear Steady-State}

In this section, the results of the GCHBM procedure and comparisons with temporal results from reference [14] are presented and discussed. Steady-state solutions for three different friction coefficients are studied. The efficiency of GCHBM is underlined as are the optimization process parameters. Finally, results relating to the evolution of frequencies and amplitude level as a function of friction coefficient are discussed. Table 2 groups the parameter values and Table 3 gathers frequency values for three different friction coefficients. 


\begin{tabular}{cccc} 
Case & Friction coefficient & $f_{1}(\mathrm{~Hz})$ & $f_{2}(\mathrm{~Hz})$ \\
\hline 1 & $\mu=0.29$ & 896.9 & 1497.1 \\
2 & $\mu=0.35$ & 903.8 & 1495.4 \\
3 & $\mu=0.4$ & 898.3 & - \\
\hline
\end{tabular}

Table 3: Parameter table

\subsubsection{Limit cycles with two unstable modes}

Figure 5 displays pseudo-periodic responses for a disc and a pad interface node for both methods at $\mu=0.29$ (with $N_{h}=3$ ).

Since the responses are pseudo-periodic due to both incommensurate frequencies, the limit cycles are no longer closed. They are delimited by a maximum amplitude shape and remain inside it. Good correlation between time integration and GCHBM is found and the relative errors on displacements and velocities are less than $5 \%$. Limit cycle evolution is complicated due to both unstable modes; consequently, the Power Spectrum Density (PSD) is plotted on Figure 6 to facilitate understanding. Since both modes participate in the nonlinear dynamic solution, the spectrum includes both fundamental frequencies, $f_{1}=897 \mathrm{~Hz}$ and $f_{2}=1497 \mathrm{~Hz}$, as well as their respective harmonics $2 f_{1}, 3 f_{1}, 2 f_{2}$ and $3 f_{2}$. Note that frequencies $f_{1}$ and $f_{2}$ of the final response are slightly different from those of the stability analysis, therefore taking them as unknowns in a GCHBM procedure is a reasonable hypothesis. Moreover, modulation frequencies are also found in the spectrum due to nonlinearities. The combinations are $2 f_{1}-f_{2}, f_{2}-f_{1}, 2 f_{2}-f_{1}, f_{1}+f_{2}, 2 f_{1}+f_{2}, f_{1}+2 f_{2}$, as indicated by equation (14) when $N_{h}=3$. These couplings have a strong effect on dynamic behavior and an example is shown in Figure 6 (b), where the amplitude level at $f_{2}-f_{1}$ is almost 100 times higher than the amplitude level of fundamental frequency $f_{2}$. Since the number of harmonics $N_{h}$ retained is equal to 3 , several frequencies were not taken into account in GCHBM, whereas the time integration shows these frequencies. For example, there is a peak at $2 f_{2}-2 f_{1}$ (Figure $6(\mathrm{a}, \mathrm{b})$ ) for the time integration but no amplitude for GCHBM.

Nonlinear behavior can be affected by all the frequency combinations if their contributions are non negligible. For Figure 6 (a) which corresponds to the pad, the contribution of $f_{2}-f_{1}$ seems to be small, but for 6 (b) which is a disc node, its power is higher than the fundamental frequency level $f_{2}$. What is more, in Figure $5(\mathrm{c}, \mathrm{d})$ differences can be clearly distinguished on the limit cycle patterns despite the fact that the maximum amplitude shape remains the same. As an illustration, Figure 7 shows the responses of the interface nodes of a disc and pad at $\mu=0.35$ (with $N_{h}=3$ ). The limit cycle shapes are still complicated and the same frequency combination can be observed in PSD in Figure 8. Nevertheless, the third harmonic of $f_{2}$ appears to be absent from the frequency spectrum for the pad node (Figure 8 (a)) while the fifth harmonic of $f_{1}$ is dominant in the frequency range $[3500-5000] \mathrm{Hz}$. Since $N_{h}=3,5 f_{1}$ is taken into account only by the time integration. In Figure 8 (b), $3 f_{2}$ is still absent but here $5 f_{1}$ no longer responds. As for $\mu=0.29$, a frequency peak at $2 f_{2}-2 f_{1}$ is taken into account only by time integration. Despite a higher friction coefficient, the amplitude of the limit cycles is lower in comparison to case 1 at $\mu=0.29$. For both cases $\mu=0.29$ and $\mu=0.35$, the disc nodes show a very low response of fundamental frequency $f_{2}$ and its harmonics compared to the first mode $f_{1}$. However, in some cases coupling frequencies have the most preponderant contribution in dynamic responses, such as $f_{2}-f_{1}$ in Figure 6 (b). Therefore keeping $f_{2}$ in the computation is essential. 


\subsubsection{Choice and influence of the harmonic number}

A common problem when dealing with harmonic balance methods is making the right choice of harmonic numbers to compute a solution. An excessively low number could lead to a poor estimation of the response, especially if strong nonlinearities exist, but choosing too many harmonics leads to unnecessarily intensive computation. Consequently, a compromise must be found. By way of example, we try to compute a nonlinear solution with the following parameters, $\left(N_{h}=2, \mu=0.29\right)$ under the same initial conditions as for $\left(N_{h}=3, \mu=0.29\right)$. It should be recalled that these initial conditions are derived from section 4.5 .

In Figure 9, the GCHBM with $N_{h}=2$ are wrong, compared to those of the time integration, as seen in Figure 10 where the harmonic components of GCHBM do not fit with those from the temporal integration. When looking at the convergence chart in Figure 11, it can be seen that $\epsilon_{1}$ has not reached a minimum (Figure $11 \mathrm{a}$ ) and the real parts $a_{1}$ and $a_{2}$ that describe $\epsilon_{2}$ (Figure $11 \mathrm{~b}$ ) are not minimized since $a_{1} \approx-6.1$ and $a_{2} \approx 2$. The solver stopped because it could not find any downward direction.

One of the reasons for this could be the fact that the restricted number of harmonics leads to an overlarge approximation of displacements and thus of the nonlinear forces used for the dynamic Jacobian computation, see equation (27). Therefore the criterion $\epsilon_{2}$ derived from the real parts associated with the complex eigenvalues applied for equation (30) would not be satisfied.

Regarding validation, Figure 12 displays the density of the power spectra when using $N_{h}=5$ for $\mu=0.29$. As expected, higher harmonics such as $4 f_{1}, 5 f_{1}, 4 f_{2}, 5 f_{2}, 2 f_{2}-2 f_{1}, 3 f_{1}-f_{2}$ and $2 f_{1}+2 f_{2}$ are found in the spectrum and the GCHBM pattern is very similar to the temporal pattern. However, the amplitude of these high frequency harmonics is negligible when compared to those found with $N_{h}=3$ and they do not appear to affect the stationary response.

Now we consider $N_{h}=2$ in a sequential continuation where we re-use the previous results as initial conditions for a solution computed with a new set of parameters. In practice, the computation is performed from $<N_{h}=3, \mu=0.345>$ to $<N_{h}=2, \mu=0.35>$. The advantage of this procedure is that we can determine whether, under better initial conditions, GCHBM is able to provide good results with fewer harmonics. The response is no, as indicated by figure 13, where the limit cycles computed by GCHBM are still far from the temporal solution. In Figure 14 it can be seen that the harmonic components $f_{1}$ and $2 f_{1}$ merge with those of the temporal integration, but $f_{2}, 2 f_{2}$ are almost absent from the spectrum. Combination frequencies such as $f_{2}-f_{1}$ or $f_{2}+f_{1}$ are also badly computed, meaning that using $N_{h}=2$ in such cases is unsatisfactory. Therefore $N_{h}=3$ seems to be a good compromise between accuracy and computation time and could be used in the following.

\subsubsection{Limit cycles with one unstable mode}

An interesting phenomenon appears when computing limit cycles for $\mu=0.4$. Figure 15 shows the results of time integration and GCHBM procedure. To enhance understanding, Figure 15(a) and (b) are for two distinct pad nodes and Figure 15(c) and (d) are for two distinct disc nodes.

Although the stability analysis shows the presence of two unstable modes for this set of parameters, the dynamic behavior of the system is only driven by the first unstable mode $f_{1}=898.3 \mathrm{~Hz}$ and the second unstable mode $f_{2}$ is totally absent in the spectrum of Figure 16. Therefore only the fundamental frequency $f_{1}$ and its harmonics $2 f_{1}$ and $3 f_{1}$ are found and coupling frequencies such as $f_{1}+f_{2}, f_{2}-$ $f_{1}$ and so on are no longer available. The inner loops in Figure 15 (a) are due to the harmonics $2 f_{1}$ and $3 f_{1}$ exacerbated by nonlinearities. Obviously, since $N_{h}=3$ was chosen for the Fourier series, harmonics higher than $3 f_{1}$ are not computed and they are found to be absent from the GCHBM spectrum. 
Nevertheless, these harmonics are not preponderant in the solution when comparing their power density to the three first harmonics.

As seen previously, the GCHBM results are close to the time integration results with a relative error on displacement less than $1 \%$. Note that GCHBM functions like a CHBM when only one unstable mode is considered in the final solution.

\subsection{Parameter evolution according to $\mu$}

In this section we present the evolution of limit cycles with associated unstable frequencies and the total amplitude energy of the system with $\mu$ varying from $\mu=0.29$ up to $\mu=0.60$ which is a part of the unstable domain. Figure 17 shows a set of limit cycles computed at different friction coefficient values. As seen previously, both modes are involved in the dynamic for a low friction coefficient with a complex limit cycle shape. When $\mu$ is above 0.36 , we found closed limit cycles, indicating that only one mode remains in the dynamic.

To study the squeal propensity of each mode, an index was defined and the following were chosen from the literature:

$$
\alpha_{j}=100 * 2 \frac{\operatorname{Re}\left(\lambda_{j}\right)}{\operatorname{Im}\left(\lambda_{j}\right)} \text { for the } j^{\text {th }} \text { mode }
$$

The ratio $\alpha_{1} / \alpha_{2}$ versus the friction coefficient $\mu$ is displayed in Figure 18. It is interesting to compare how the ratio behaves with $\mu$ and its effects on the dynamic steady-state. When looking at the first part of the curve, i.e. for $0.29<\mu<0.36$, a very steep slope can be seen, but from $\mu=0.36$ ratio $\alpha_{1} / \alpha_{2}$ remains almost constant with respect to $\mu$. When looking at Figure 17 it can be seen that the transition between a pseudo-periodic response to a periodic response also occurs at around $\mu=0.36$. It seems as if there is an analogy between the transition of the index ratios of both modes and their availability or non-availability in the dynamic response. The ratio increases until the first mode $m_{1}$ replaces the second mode $m_{2}$ and remains constant when $m_{1}$ is the only mode providing a response.

As can be seen, a squeal propensity index higher by tenfold is found for the $1^{\text {st }}$ mode compared to the $2^{\text {nd }}$ mode at $\mu=0.4$. The energy of the first mode appears to replace the second mode even if the latter is present in the stability analysis.

To facilitate understanding, Figure 19 (a) shows frequencies for $\mu$ varying from $\mu=0.29$ to $\mu=$ 0.36 where both modes are unstable. The frequencies are friction coefficient dependent, and while $f_{2}$ decreases as $\mu$ increases, the slope of $f_{1}$ curve is positive in this range. Nevertheless, $f_{2}$ disappears from the dynamic steady-state at around $\mu=0.36$ as can be seen in Figure 19 (b) where only the first unstable mode $f_{1}$ is present in the limit cycle in the range between $\mu=0.36$ to $\mu=0.68$. Frequency $f_{1}$ follows a negative slope regarding $\mu$, it decreases from $905 \mathrm{~Hz}$ at $\mu=0.36$ to $860 \mathrm{~Hz}$ at $\mu=0.68$.

Figure 19 (c) shows the total amplitude energy of the whole system for $\mu$ ranging from 0.29 to 0.68 . In the first part, until $\mu=0.36$ where both modes are unstable, the energy decreases and a minimum is found when $f_{2}$ vanishes from the solution. From $\mu=0.36$, the energy of the system increases with a quadratic form with respect to $\mu$. The first unstable mode $f_{1}$ seems to act as energy pulsating in the active range of the second mode $f_{2}$ before it disappears from the solution. As described above, the friction coefficient not only modifies the amplitude of the dynamic behavior of autonomous systems, but it also influences their frequencies. This dependency between friction and frequency could have an effect on neighboring modes by exciting them. Consequently, they may participate in the final dynamic solution. 


\subsection{Convergence and Computation Time}

Convergence problems stem from many sources when attempting optimization. The main problems come from initial estimates made far from the target solution. Here convergence is achieved with the GCHBM algorithm because a set of conditions considered as optimized is used. For each iteration, Figure 20 displays the norm of the real parts (a), both optimized frequencies (b), and the norm of the residue (c). More precisely, frequency plot (b) corresponds to the difference between the optimized frequencies and those obtained by a stability analysis.

The total number of iterations is $N_{i t e r}=35$. The real parts both tend to zero at the end of the optimization process, as does the norm of the residue. A net difference between the initial frequencies obtained by the stability analysis and those at the end of optimization is shown. For the first mode $f_{1}$, the frequency evolution is about $20 \mathrm{~Hz}$. GCHBM enhances computation time, which falls to about 3 hours, 20 minutes, whereas time integration needs about 10 hours to obtain the dynamic steady-state. A better convergence result could be obtained by trying a new initial starting point or by changing the tolerance values $\epsilon_{1}$ and $\epsilon_{2}$, since Figure 20 clearly shows that both real parts and frequencies as well as the norm of the residue have almost converged at iteration 25 . This would significantly reduce total computation time. Here we have chosen $\epsilon_{1}=\epsilon_{2}=0.01$. It should be noted that these convergence results stem from the very first computation. When using another set of parameters, such as a change of friction coefficient, the results computed previously are injected as an initial starting point and computation is performed. Hence the number of iterations and thus computation time are considerably reduced, since only a few iterations are required to converge to the final solution. In general, a new steady-state solution can be computed in less than 20 minutes.

\section{Conclusion}

In this paper we develop a new method called GCHBM able to compute the dynamic steady-states of autonomous systems such as disc brake squeal in the case where stability analysis reveals large numbers of unstable modes. The computed solutions are either pseudo-periodic if at least two unstable modes generate vibrations, or periodic if dynamic behavior is driven by only one unstable mode. When at least two unstable modes vibrate, the stationary dynamic responses become pseudo-periodic and the cycle plotted in the displacement-velocity coordinates is no longer closed. Particular care regarding the total harmonic number must be considered to avoid missing important coupling frequencies or even the non convergence of the solver. Finally, GCHBM is well suited for brake squeal analysis when a large number of unstable modes are taken into account. It could help brake designers by computing the amplitudes of limit cycles for sets of parameters faster than with time integration. Frequency responses are analyzed and modal behavior is better understood. For example, it detects the absence of the second mode $f_{2}$ at $\mu=0.36$ although the stability analysis predicts two unstable modes. Dynamic behavior can be computed for numerous parameter sets and an optimal area can be found easily. For example, the lowest dynamic response of our model occurs at $\mu=0.36$ in the unstable area, the point at which the second mode $f_{2}$ disappears from the dynamic response.

\section{Acknowledgements}

Jean-Jacques Sinou gratefully acknowledges the financial support of the French National Research Agency through the Young Researcher program ANR-07-JCJC-0059-01-CSD 2. 


\section{References}

[1] Kinkaid, N., O’Reilly, O., and Papadopoulos, P., 2003. "Automotive disc brake squeal". Journal of Sound and Vibration, 267, pp. 105-166.

[2] Ouyang, H., Nack, W., Yuan, Y., and Chen, F., 2005. "Numerical analysis of automotive disc brake squeal : a review". International Journal of Vehicle Noise and Vibration, 1(3/4), pp. 207-231.

[3] Sinclair, D., and Manville, N.-J., 1955. "Frictional vibration". Journal of Applied Mechanics, pp. 207-213.

[4] Spurr, R.-T., 1961-1962. "A theory of brake squeal". Proceedings of the Institution of Mechanical Engineers, 1, pp. 33-40.

[5] Hoffmann, N., Fisher, M., Allgaier, R., and Gaul, L., 2002. "A minimal model for studying properties of the mode-coupling type instability in friction induced oscillations". Mechanics Research Communications, 29, pp. 197-205.

[6] Sinou, J.-J., Thouverez, F., and Jezequel, L., 2004. "Methods to reduce non-linear mechanical systems for instability computation". Archives of Computational Methods in Engineering - State of the art reviews, 11(3), pp. 257-344.

[7] Moirot, F., 1998. "Etude de la stabilité d'un équilibre en présence de frottement de coulomb, application au crissement des freins à disque". $\mathrm{PhD}$ thesis, Ecole Polytechnique.

[8] Ouyang, H., Mottershead, J., Brookfield, D., James, S., and Cartmell, M., 2000. "Methodology for the determination of dynamic instabilities in a car disc brake". International Journal of Vehicle Design, 23(3), pp. 241-262.

[9] Massi, F., Baillet, L., Giannini, O., and Sestieri, A., 2007. "Linear and non-linear numerical approaches”. Mechanical Systems and Signal Processing, 21, pp. 2374-2393.

[10] AbuBakar, A., and Ouyang, H., 2006. "Complex eigenvalue analysis and dynamic transient analysis in predicting disc brake squeal". International Journal of Vehicle Noise and Vibration, 2(2), pp. 143-155.

[11] Lorang, X., Foy-Margiocchi, F., Nguyen, Q., and Gautier, P., 2006. "Tgv disc brake squeal”. Journal of Sound and Vibration, 293, pp. 735-746.

[12] Groll, G.-V., and Ewins, D.-J., 2001. "The harmonic balance method with arclength continuation in rotor/stator contact problems". Journal of Sound and Vibration, 241(2), pp. 223-233.

[13] Coudeyras, N., Sinou, J.-J., and Nacivet, S., 2008. "A new treatment for predicting the self-excited vibrations of nonlinear systems with frictional interfaces: The chbm". Journal of Sound and Vibration, 319, pp. 1175-1199.

[14] Sinou, J.-J., Coudeyras, N., and Nacivet, S., 2008. "Study of the nonlinear stationary dynamic of single and multi instabilities for disc brake squeal". International Journal of Vehicle Design, 51(1-2), pp. 207-222.

[15] Craig, R.-J., and Bampton, M., 1968. "Coupling of substructures for dynamic analyses". American Institute of Aeronautics and Astronautics - Journal, 6(7), pp. 1313-1319. 
[16] Fritz, G., Sinou, J.-J., Duffal, J.-M., and Jezequel, L., 2007. "Effects of damping on brake squeal coalescence patterns-application on a finite element model". Mechanics Research Communications, 34, pp. 181-190.

[17] Kim, Y.-B., and Choi, S.-K., 1997. "A multiple harmonic balance method for the internal resonant vibration of a non-linear jeffcot rotor". Journal of Sound and Vibration, 208(3), pp. 745-761.

[18] Schilder, F., Vogt, W., Schreiber, S., and Osinga, H.-M., 2005. Fourier methods for quasi-periodic oscillations. Tech. rep., Bristol Centre for Applied Nonlinear Mathematics.

[19] Chua, L.-O., and Ushida, A., 1981. "Algorithm for computing almost periodic steady-state response of nonlinear systems to multiple input frequencies". IEEE Transactions on Circuits and Systems, 28(10), pp. 953-971.

[20] Ushida, A., and Chua, L.-O., 1984. "Frequency-domain analysis of nonlinear circuits driven by multi-tone signals". IEEE Transactions on Circuits and Systems, 31(9), pp. 766-778.

[21] Cameron, T. M., and Griffin, J. H., 1989. "An alternating frequency/time domain method for calculating the steady-state response of nonlinear dynamic systems". Journal of Applied Mechanics, 56(1), pp. 149-154.

[22] Iwan, W.-D., 1973. "A generalization of the concept of equivalent linearization". International Journal of Non-Linear Mechanics, 8, pp. 279-287.

[23] Iwan, W.-D., and A.-B., M., 1979. "Equivalent linearization for systems subjected to non-stationary random excitation". International Journal of Non-Linear Mechanics, 15, pp. 71-82.

[24] Hanh, E., and Chen, P., 1994. "Harmonic balance analysis of general squeeze film damped multidegree-of-freedom rotor bearing systems". Journal of Tribology, 116, p. 499-507.

[25] Sinou, J.-J., Thouverez, F., and Jezequel, L., 2006. "Stability analysis and non-linear behavior of structural systems using the complex non-linear analysis (cnlma)". Computers and Structures, 84, pp. 1891-1905. 


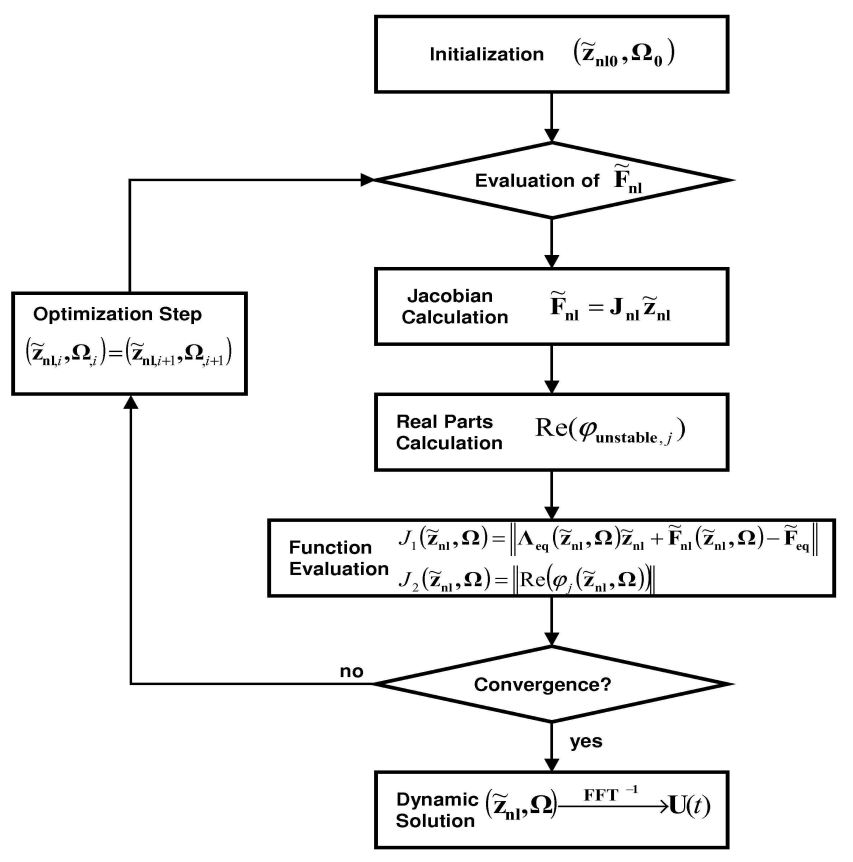

Figure 3: General algorithm for the GCHBM procedure 


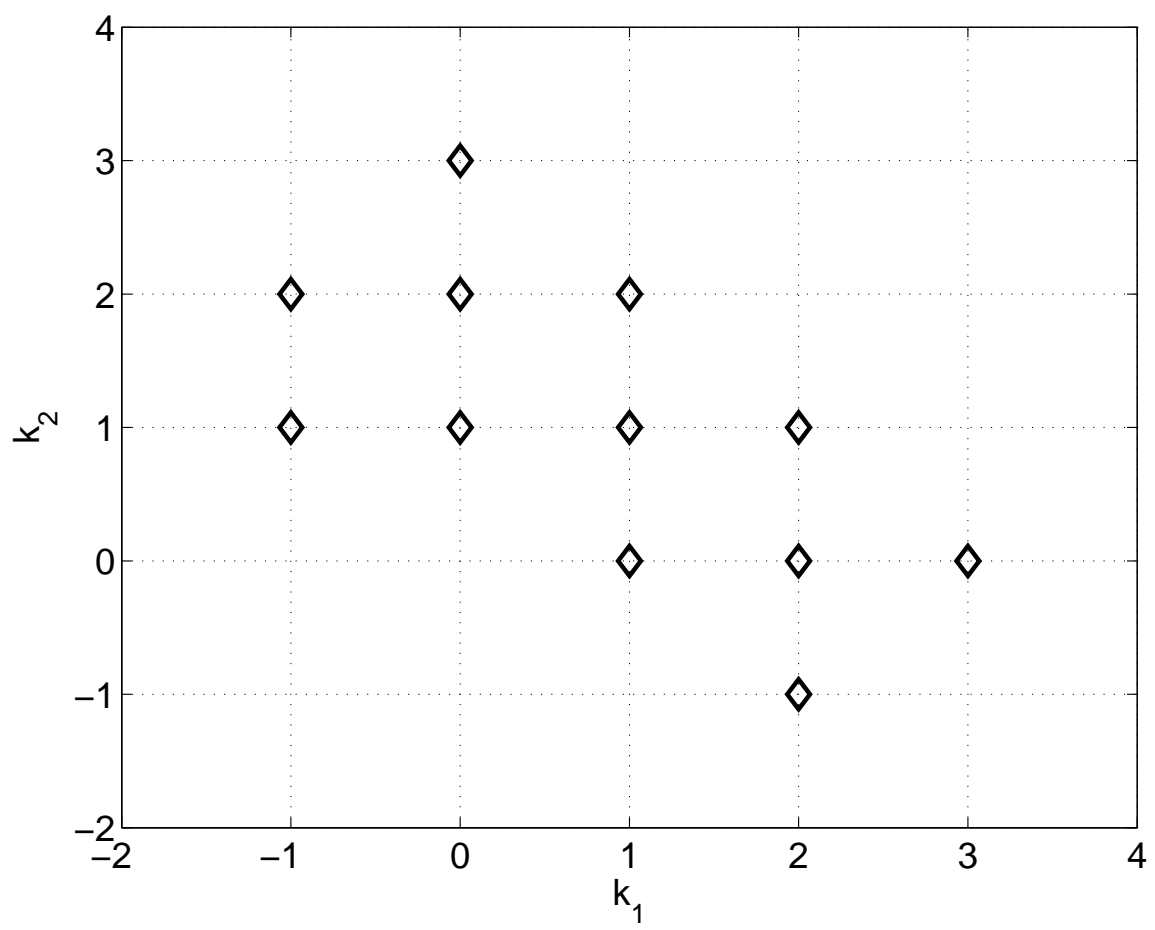

Figure 4: Harmonics components for $p=2$ and $N_{h}=3$ 
$\mathrm{a}$

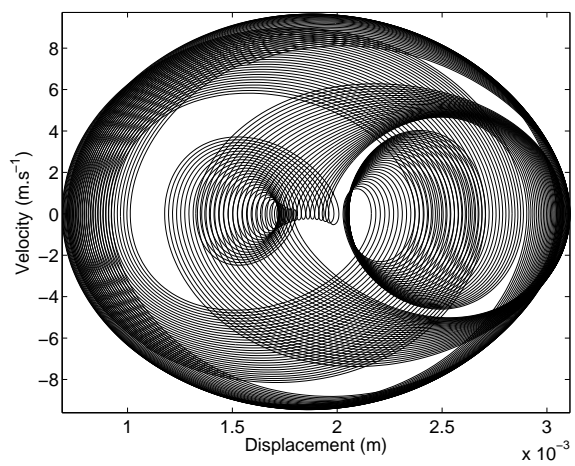

c

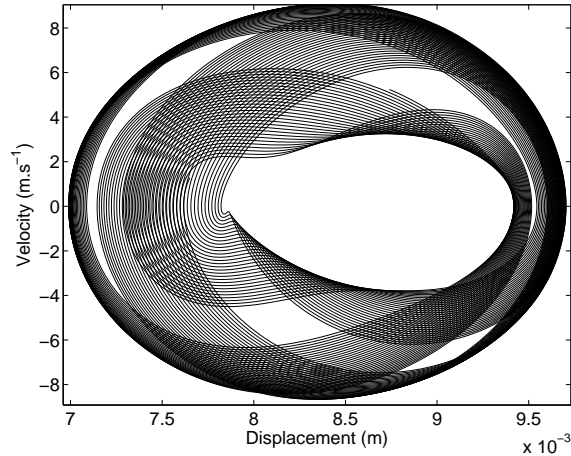

$\mathrm{e}$

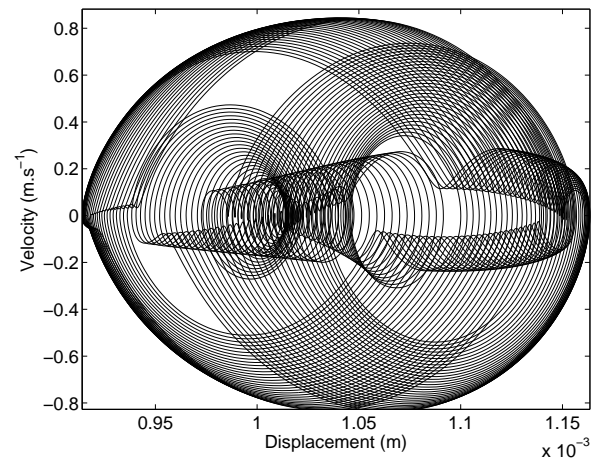

$\mathrm{g}$

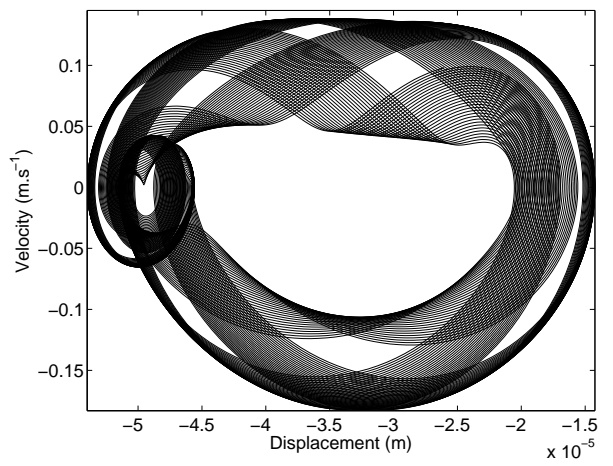

b

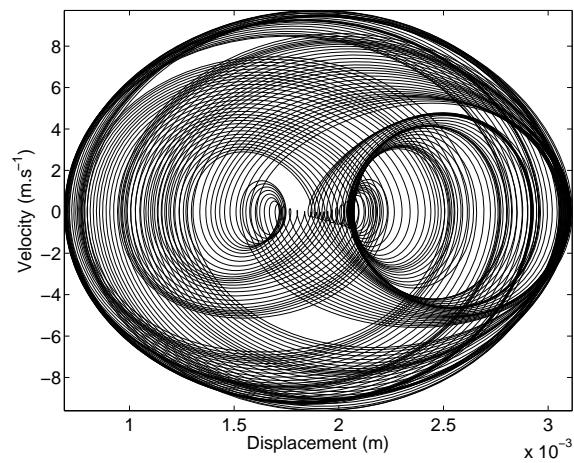

d

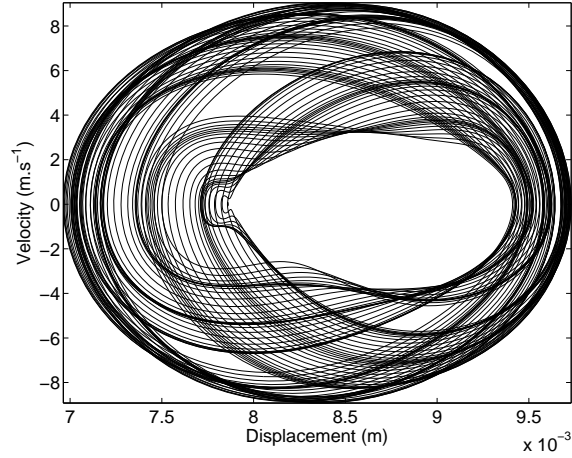

f

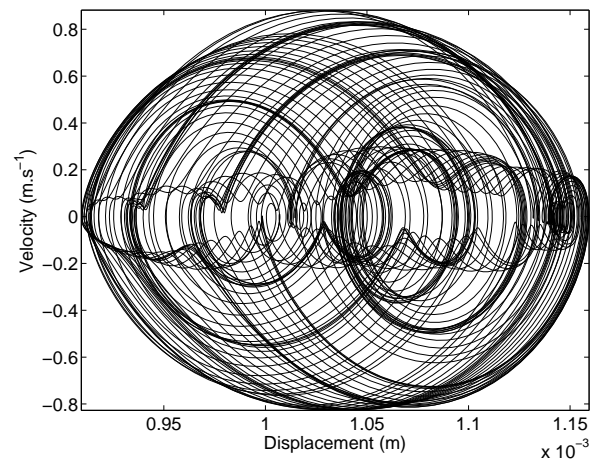

h

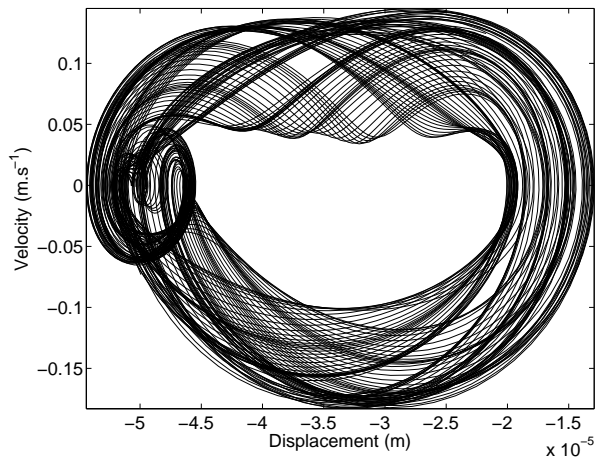

Figure 5: Limit cycles for $\mu=0.29$. (a,b,c,d) Pad node: (a,c) GCHBM with $N_{h}=3$, (b,d) Time Integration; (e,f,g,h) Disc node: (e,g) GCHBM with $N_{h}=3$, (f,h) Time Integration 
a

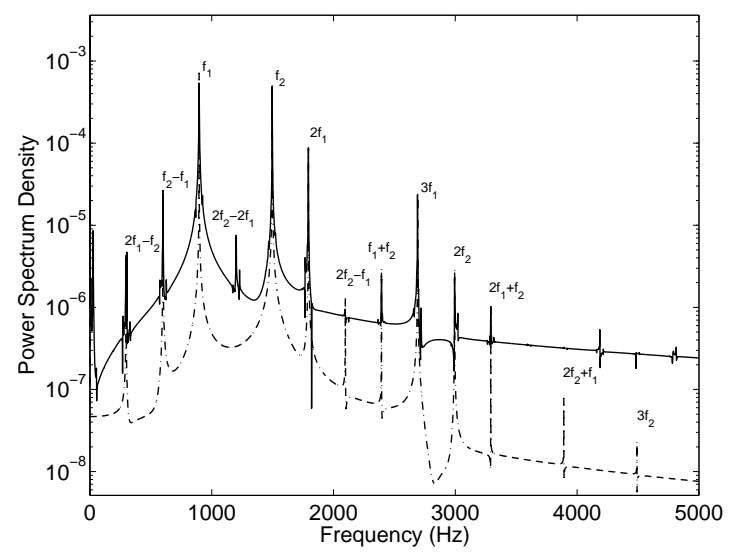

b

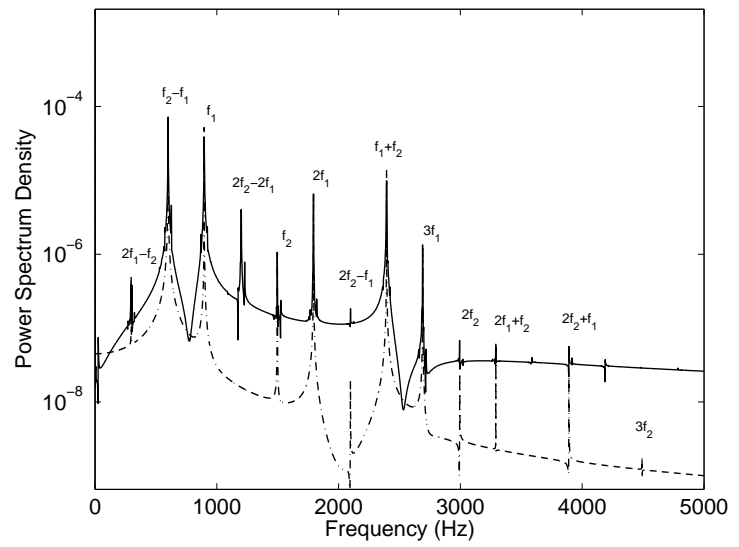

Figure 6: Power Spectrum Density for $\mu=0.29$ with $N_{h}=3$. (a) Pad node: - - GCHBM, - Time Integration; (b) Disc node: - - GCHBM, - Time Integration 
$\mathrm{a}$

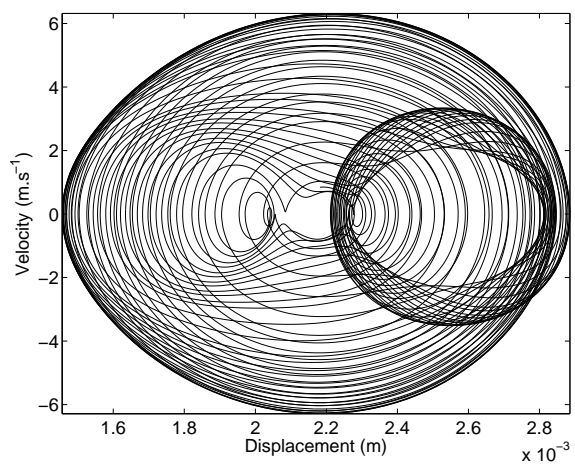

c

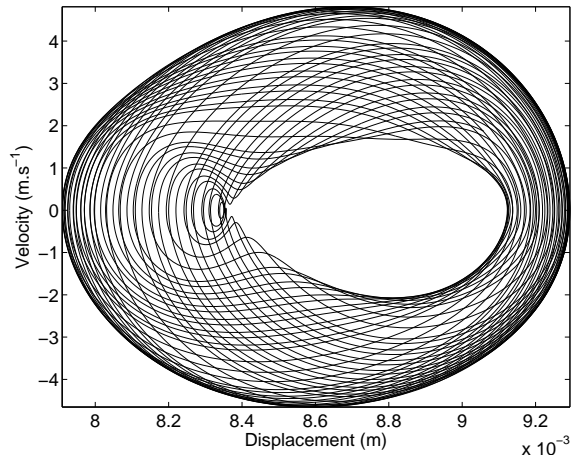

$\mathrm{e}$

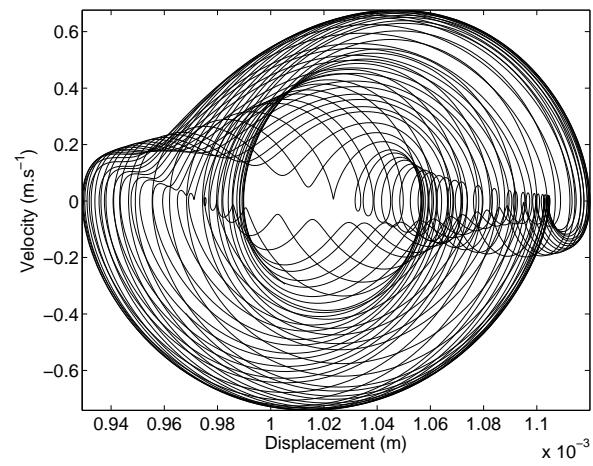

$\mathrm{g}$

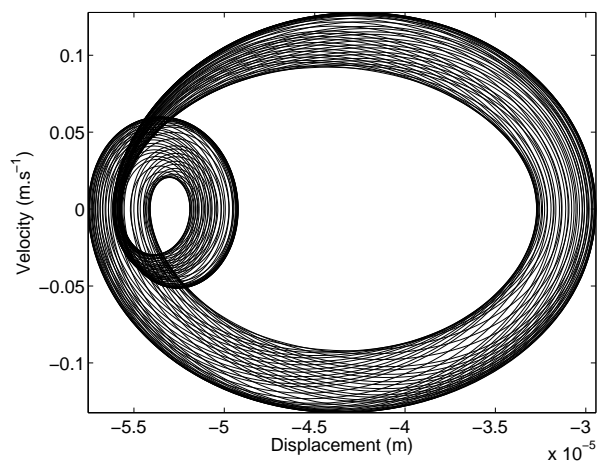

b

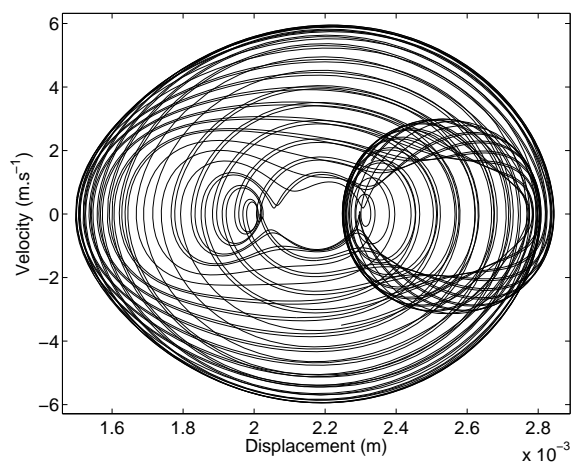

d

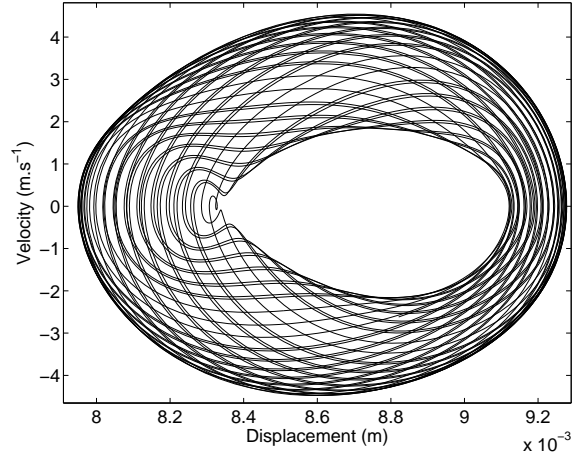

f

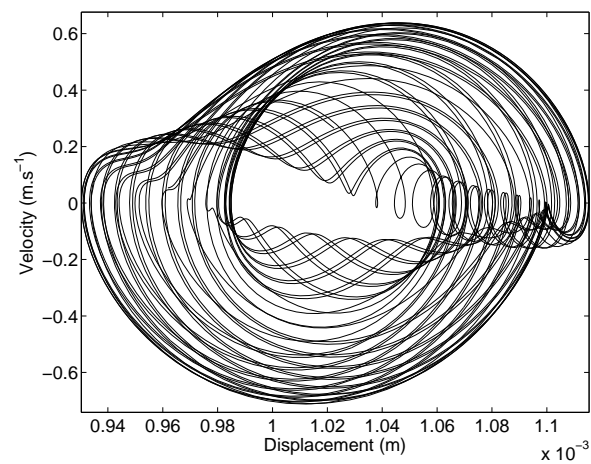

$\mathrm{h}$

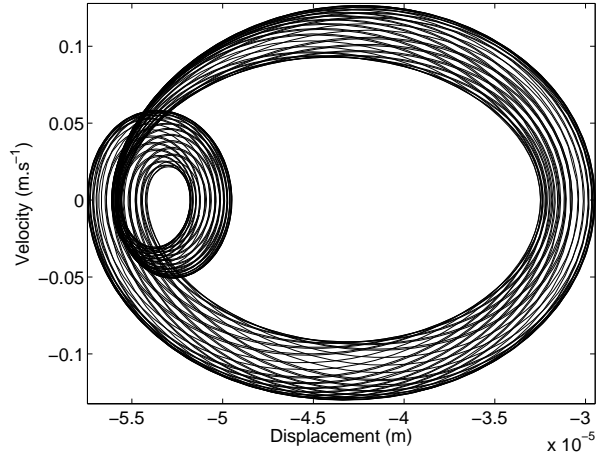

Figure 7: Limit cycles for $\mu=0.35$. (a,b,c,d) Pad node: (a,c) GCHBM with $N_{h}=3$, (b,d) Time Integration; (e,f,g,h) Disc node: (e,g) GCHBM with $N_{h}=3$, (f,h) Time Integration 
a

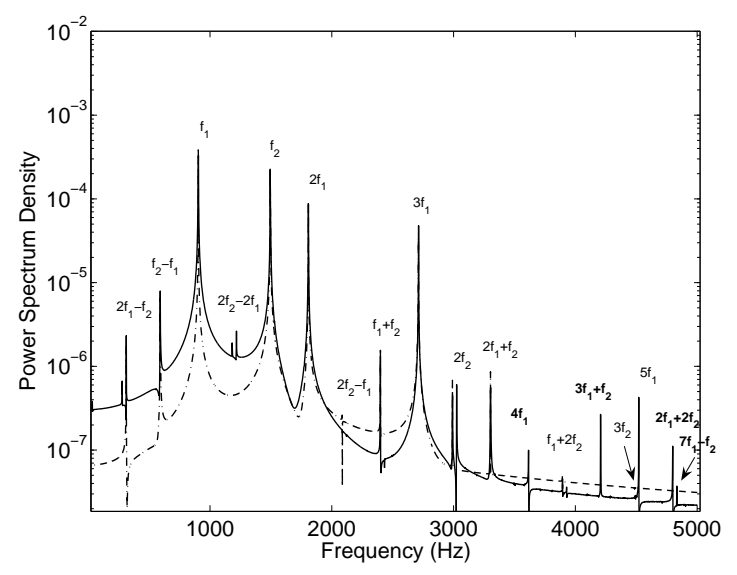

b

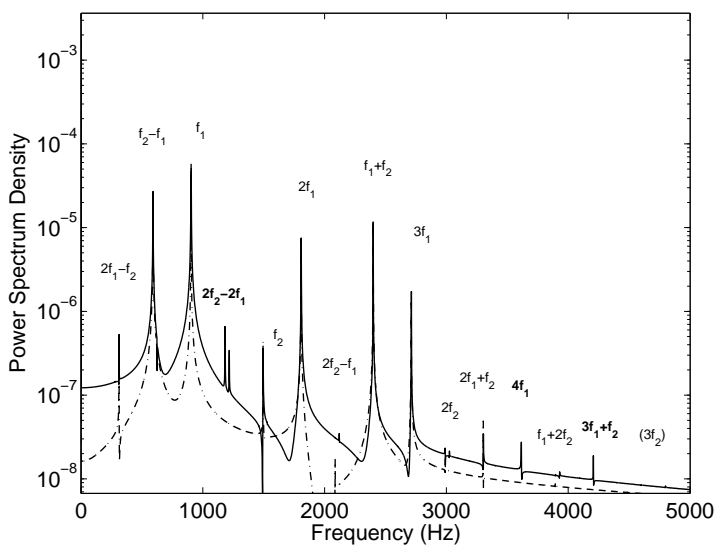

Figure 8: Power Spectrum Density for $\mu=0.35$ with $N_{h}=3$. (a) Pad node: - - GCHBM, - Time Integration; (b) Disc node: - - GCHBM, - Time Integration 
a

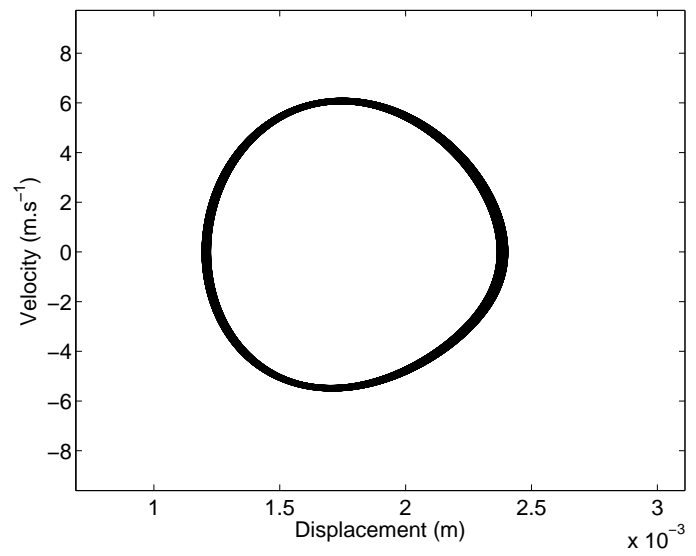

c

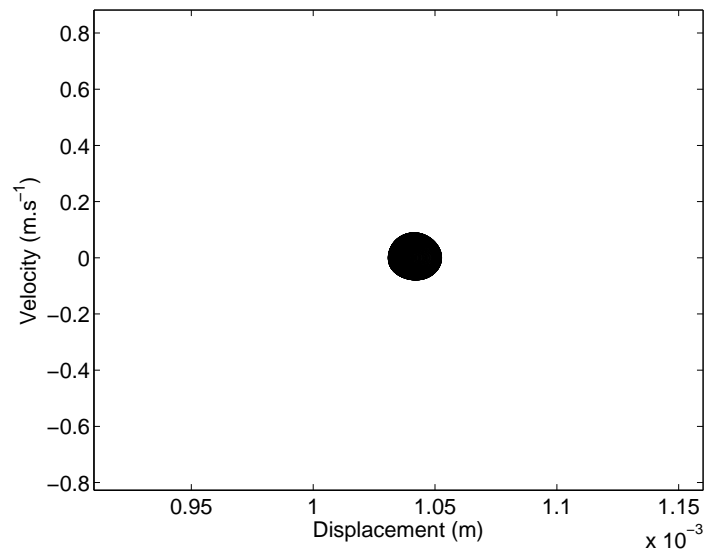

b

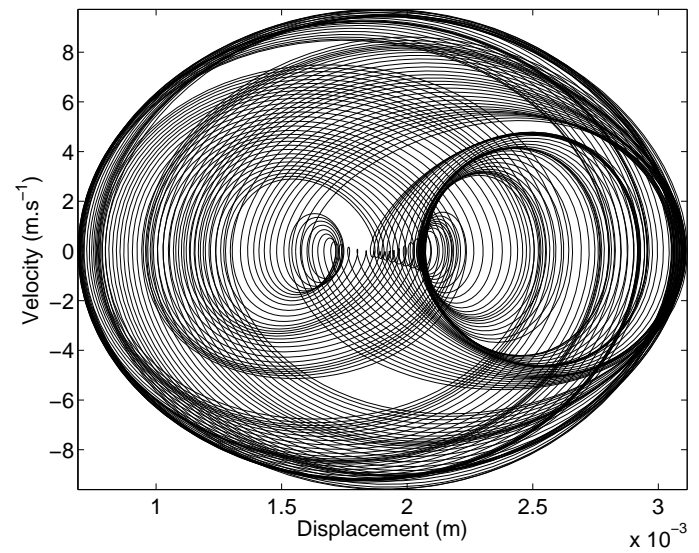

d

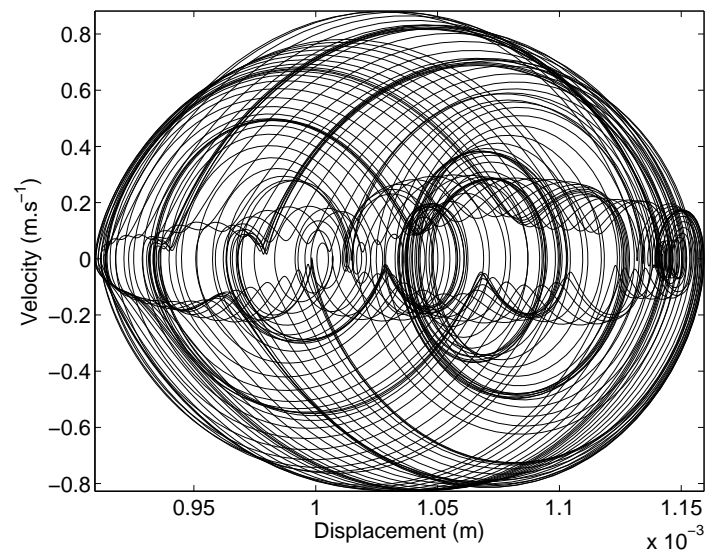

Figure 9: Limit cycles for $\mu=0.29$ and $N_{h}=2$. (a,b) Pad node: (a) GCHBM, (b) Time Integration; (c,d) Disc node: (c) GCHBM, (d) Time Integration 
a

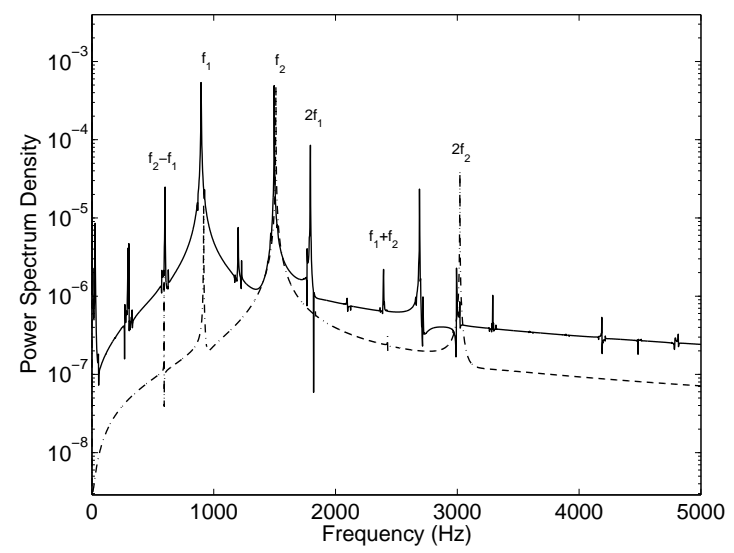

b

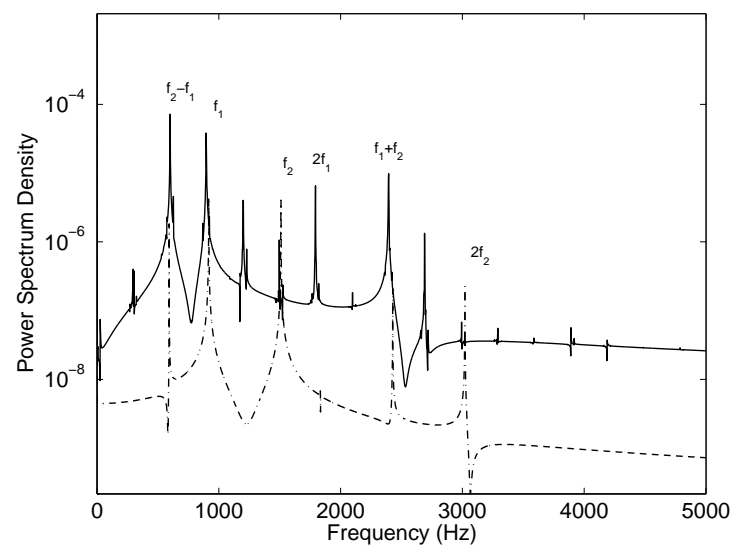

Figure 10: Power Spectrum Density for $\mu=0.29$ and $N_{h}=2$. (a) Pad node: - - GCHBM, - Time Integration; (b) Disc node: - - GCHBM, — Time Integration

a

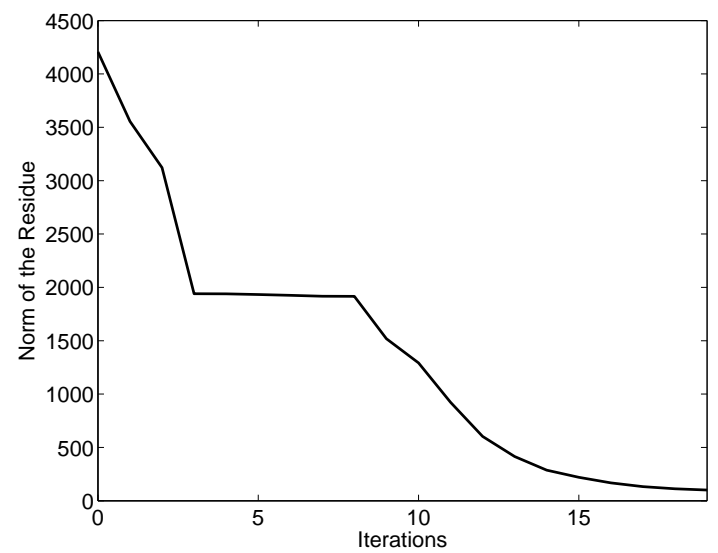

b

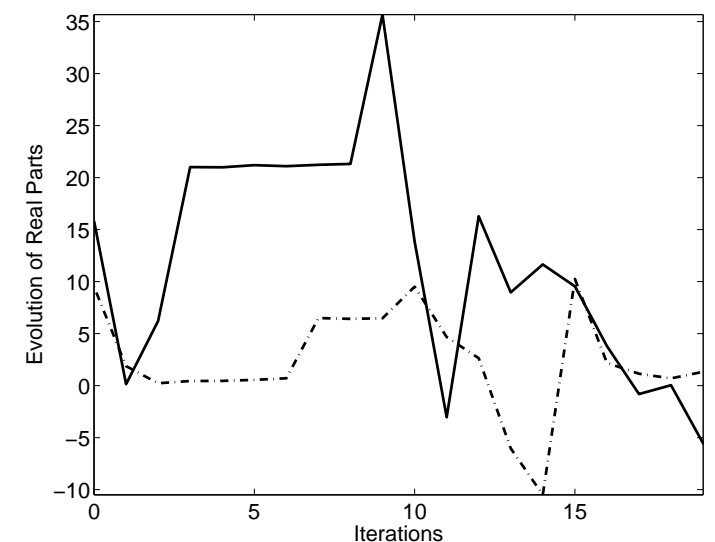

Figure 11: Evolution of parameters with iterations ( $N_{h}=2, \mu=0.29$ ). (a) Norm of Residue; (b) Real Parts: $-1^{\text {st }}$ mode, -.- $2^{\text {nd }}$ mode 
a

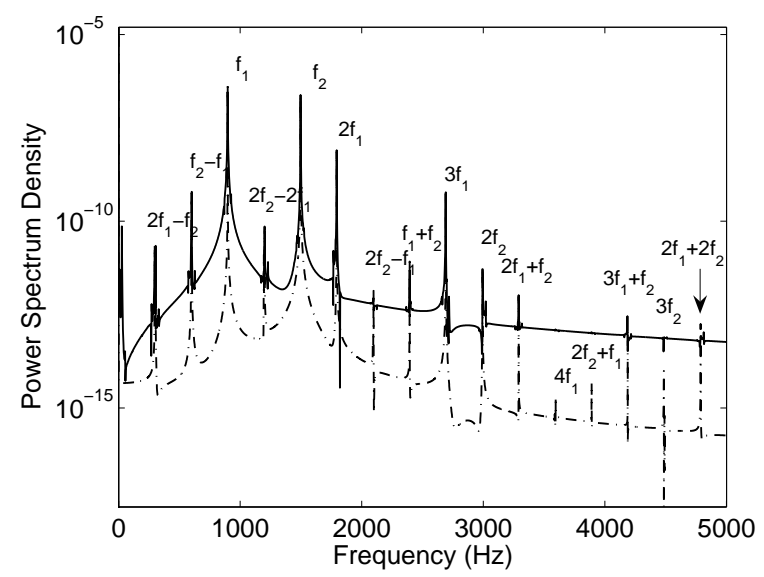

b

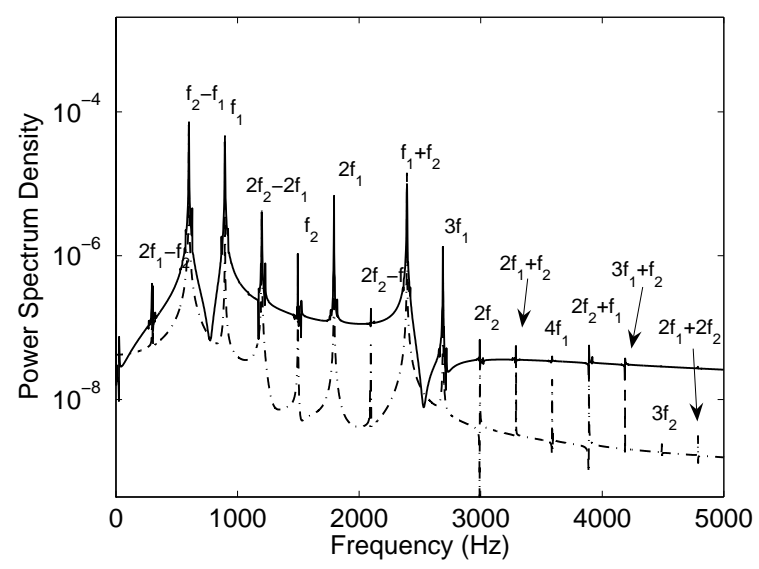

Figure 12: Power Spectrum Density for $\mu=0.29$ with $N_{h}=5$. (a) Pad node: - - GCHBM, - Time Integration; (b) Disc node: - - GCHBM, - Time Integration 
a

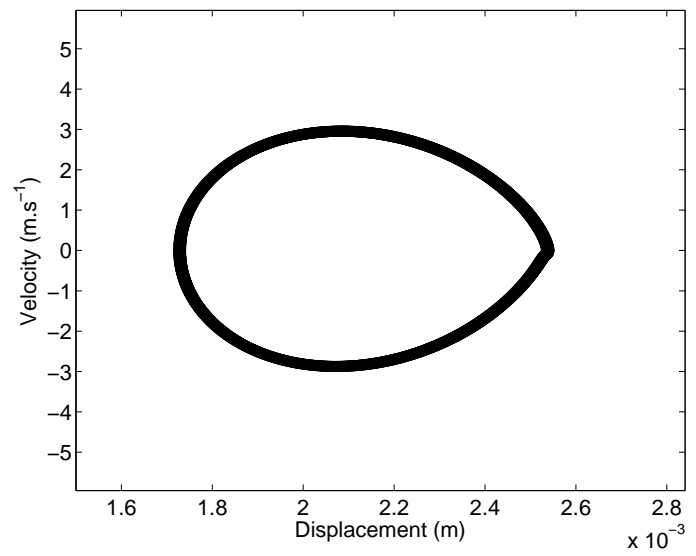

c

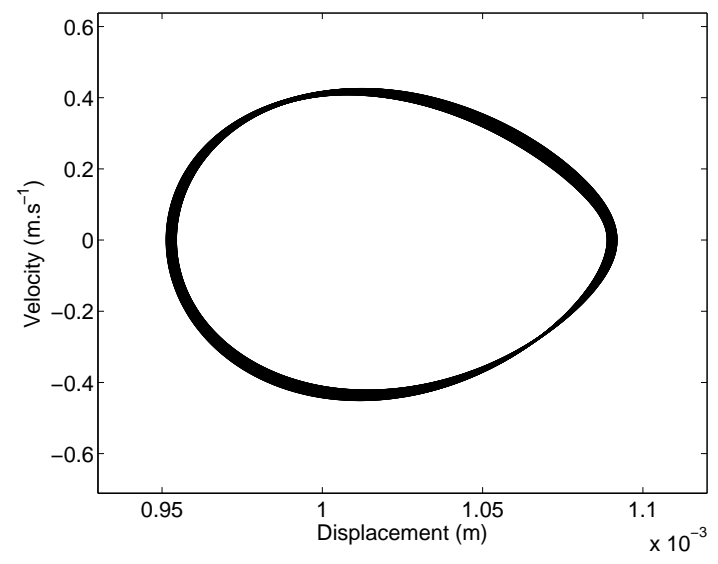

b

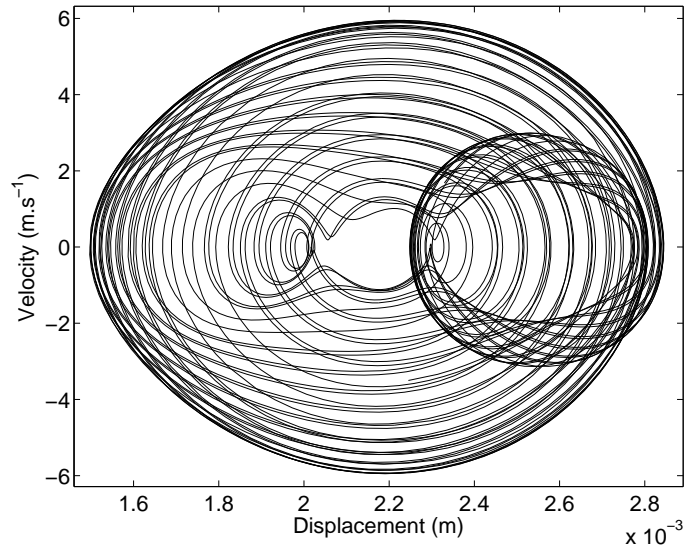

d

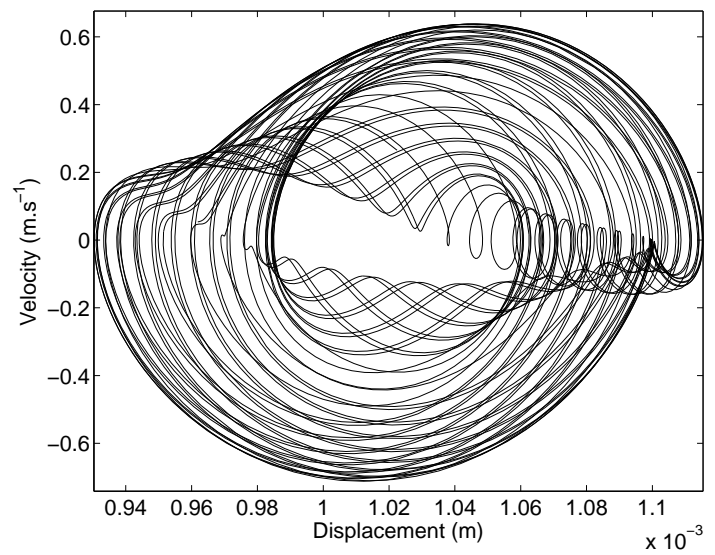

Figure 13: Limit cycles for $\mu=0.35$ and $N_{h}=2$. (a,b) Pad node: (a) GCHBM, (b) Time Integration; (c,d) Disc node: (c) GCHBM, (d) Time Integration 

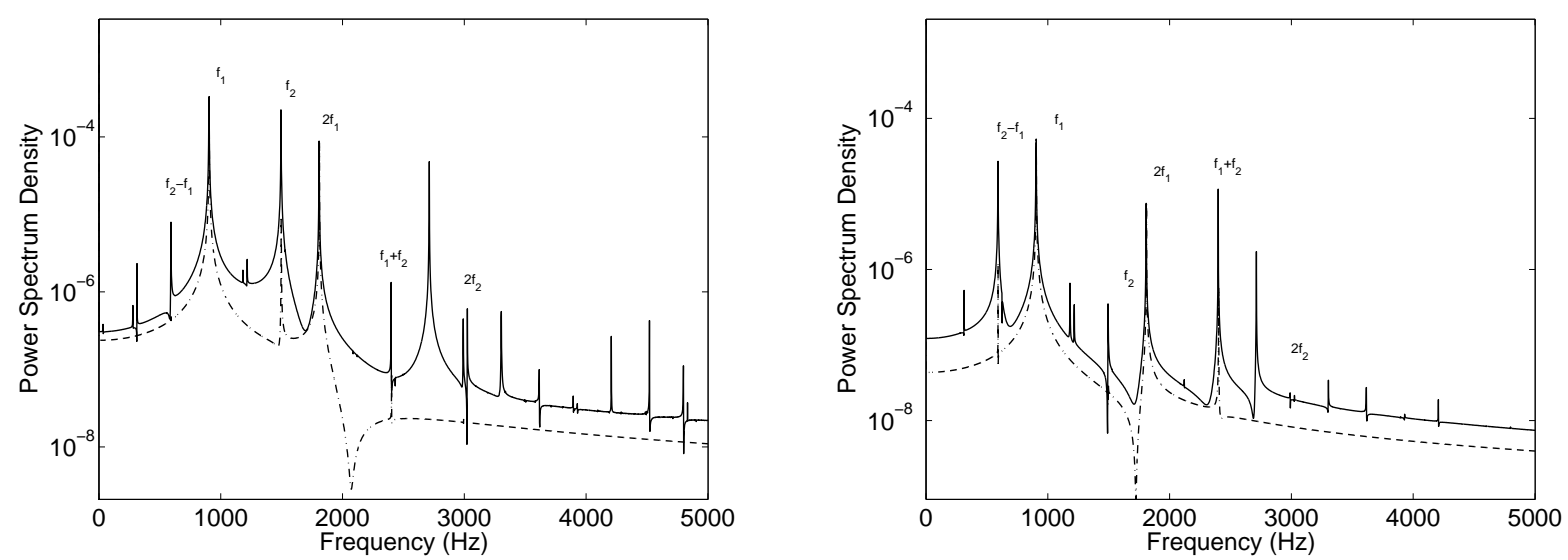

Figure 14: Power Spectrum Density for $\mu=0.35$ and $N_{h}=2$. (a) Pad node: - - GCHBM, - Time Integration; (b) Disc node: - - GCHBM, - Time Integration 
a

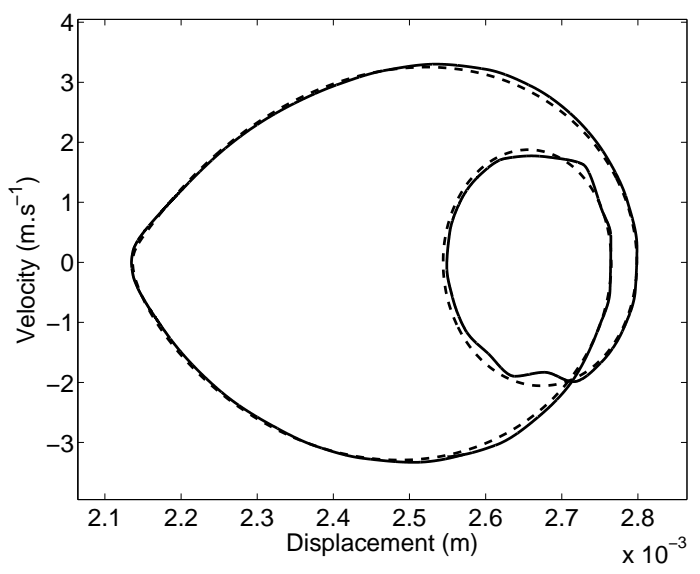

c

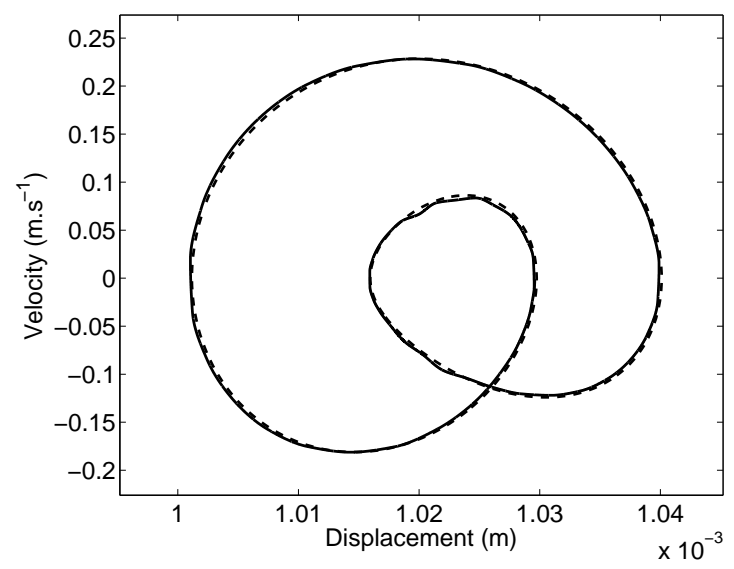

b

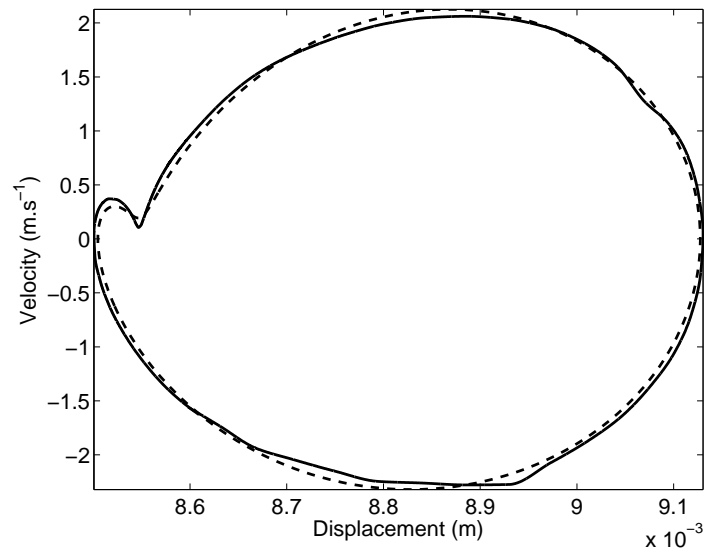

d

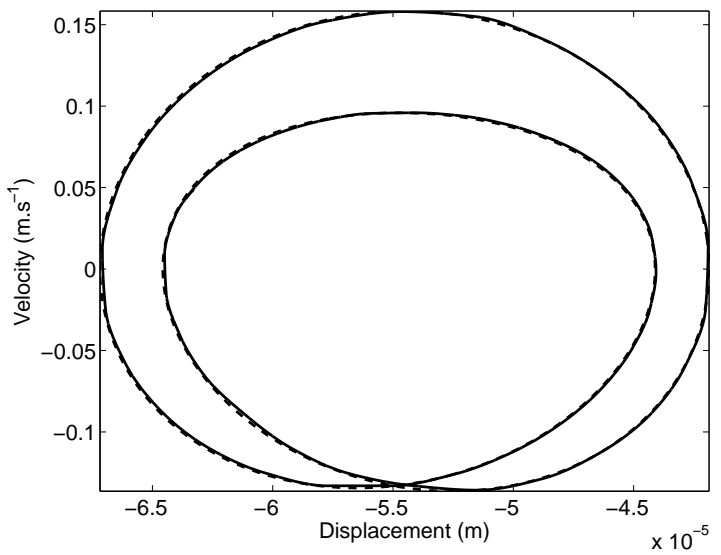

Figure 15: Limit cycles for $\mu=0.40$. (a,b) Pad node: - - GCHBM, - Time Integration; (c,d) Disc node:- - GCHBM, - Time Integration 

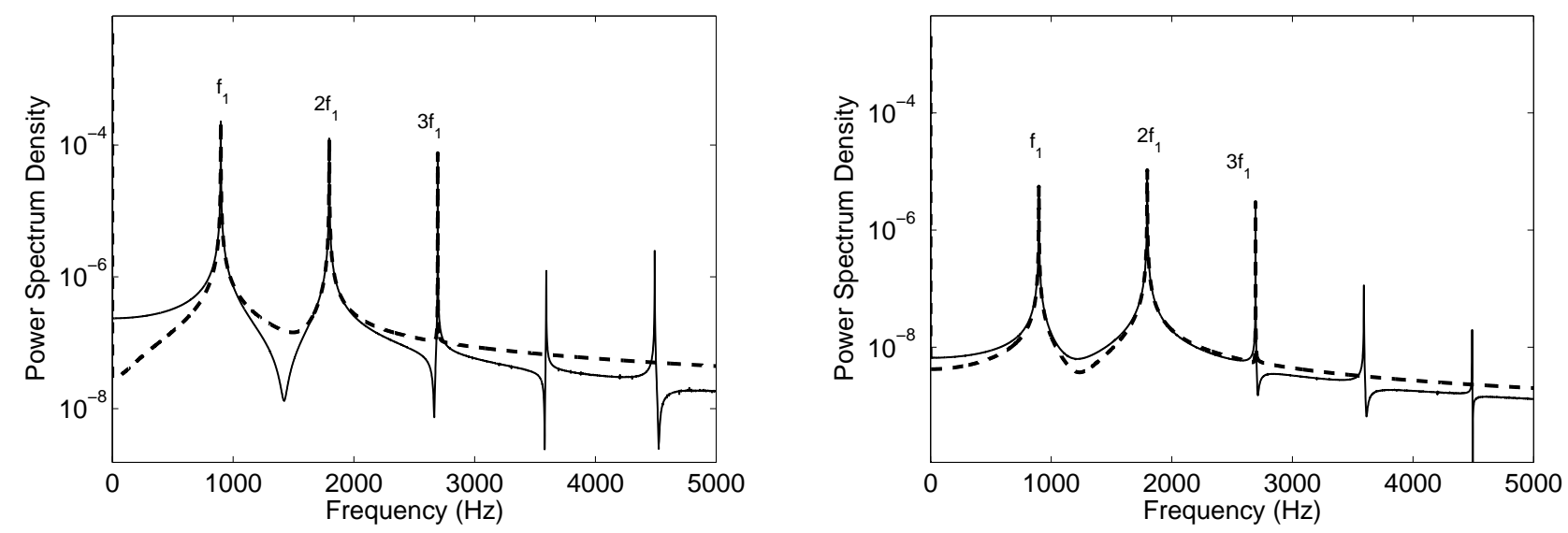

Figure 16: Power Spectrum Density for $\mu=0.40$. (a) Pad node: - - GCHBM, - Time Integration; (b) Disc node: - - GCHBM, - Time Integration 


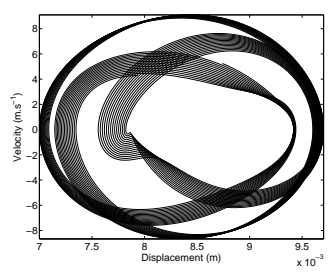

$\mu=0.29$

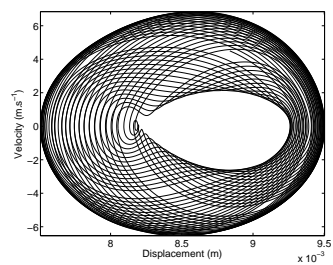

$\mu=0.33$

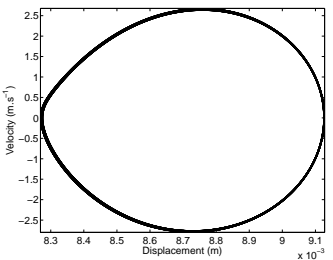

$\mu=0.36$

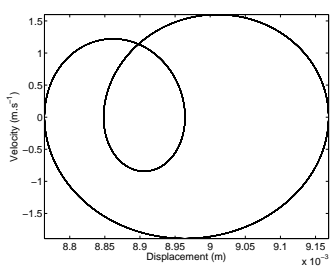

$\mu=0.48$

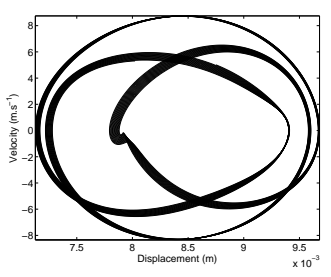

$\mu=0.30$

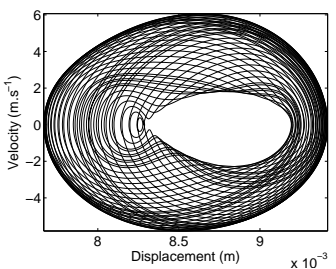

$\mu=0.34$

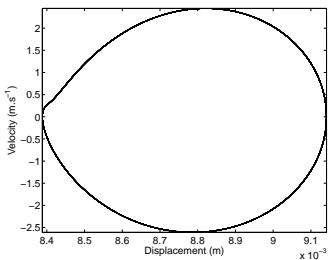

$\mu=0.38$

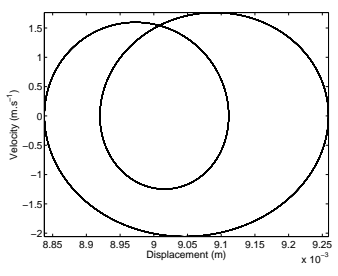

$\mu=0.52$

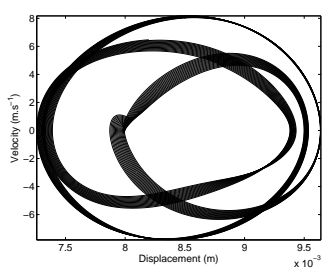

$\mu=0.31$

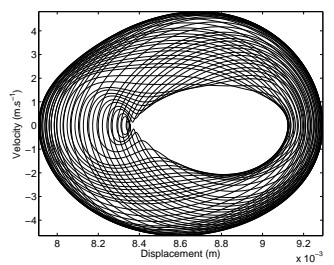

$\mu=0.35$

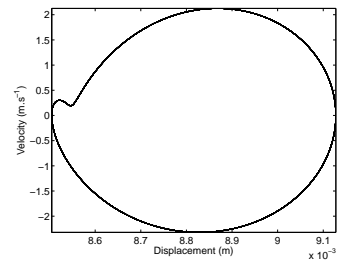

$\mu=0.40$

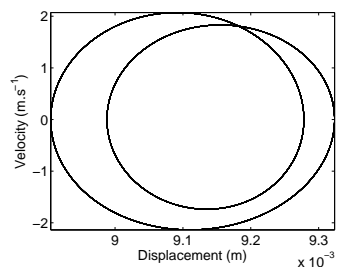

$\mu=0.56$

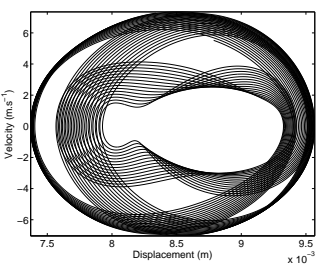

$\mu=0.32$

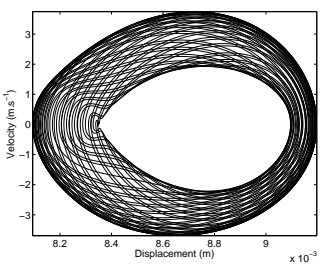

$\mu=0.355$

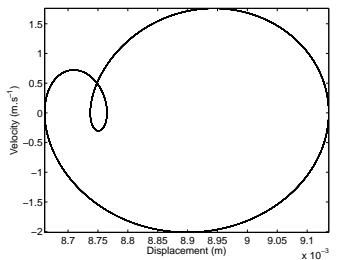

$\mu=0.44$

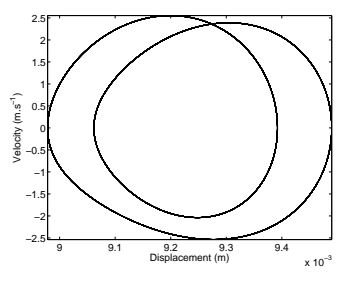

$\mu=0.60$

Figure 17: Limit cycles from the GCHBM with varying friction coefficient $\mu$ 


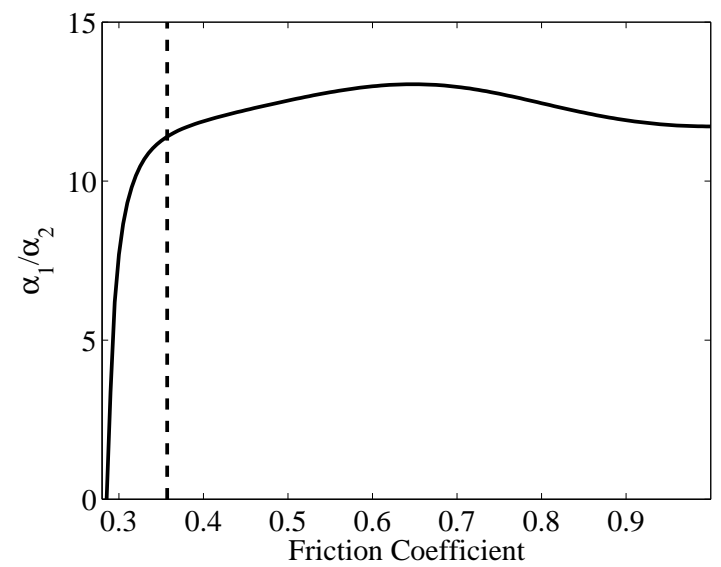

Figure 18: Propensity index ratio $\alpha_{1} / \alpha_{2}$ versus $\mu$. The dotted line indicates the transition from two to one unstable mode in the dynamic steady-state. 
a
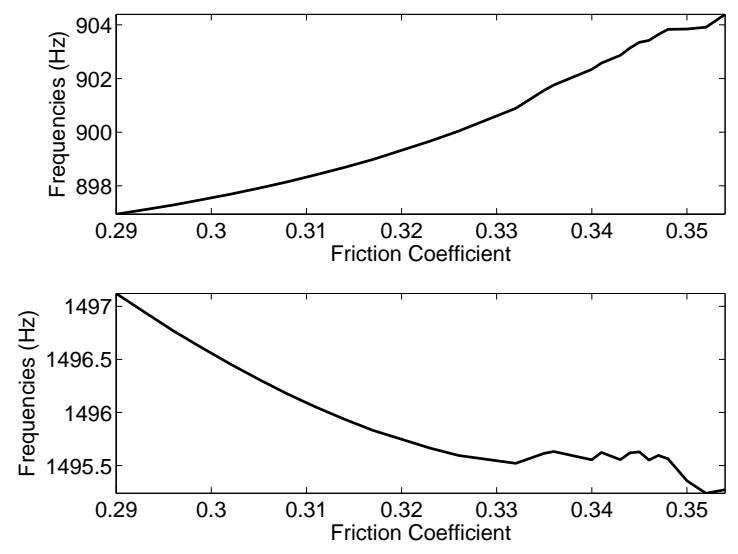

c

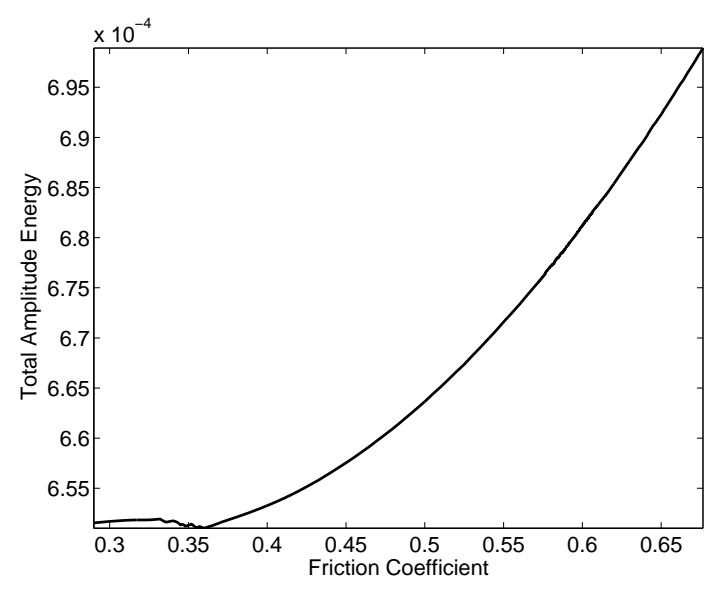

b

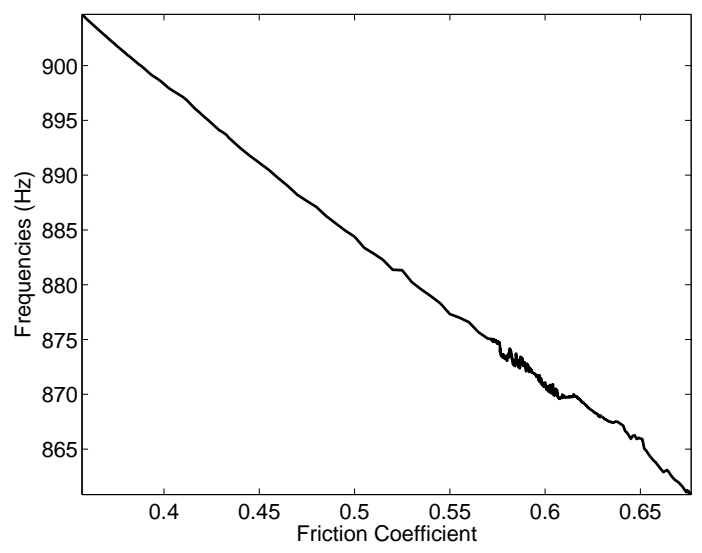

Figure 19: Evolution of parameters with $\mu$. (a) Both unstable modes, $0.29<\mu<0.36$; (b) First unstable mode $0.36<\mu<0.68$; (c) Total amplitude energy, $0.29<\mu<0.68$ 
b
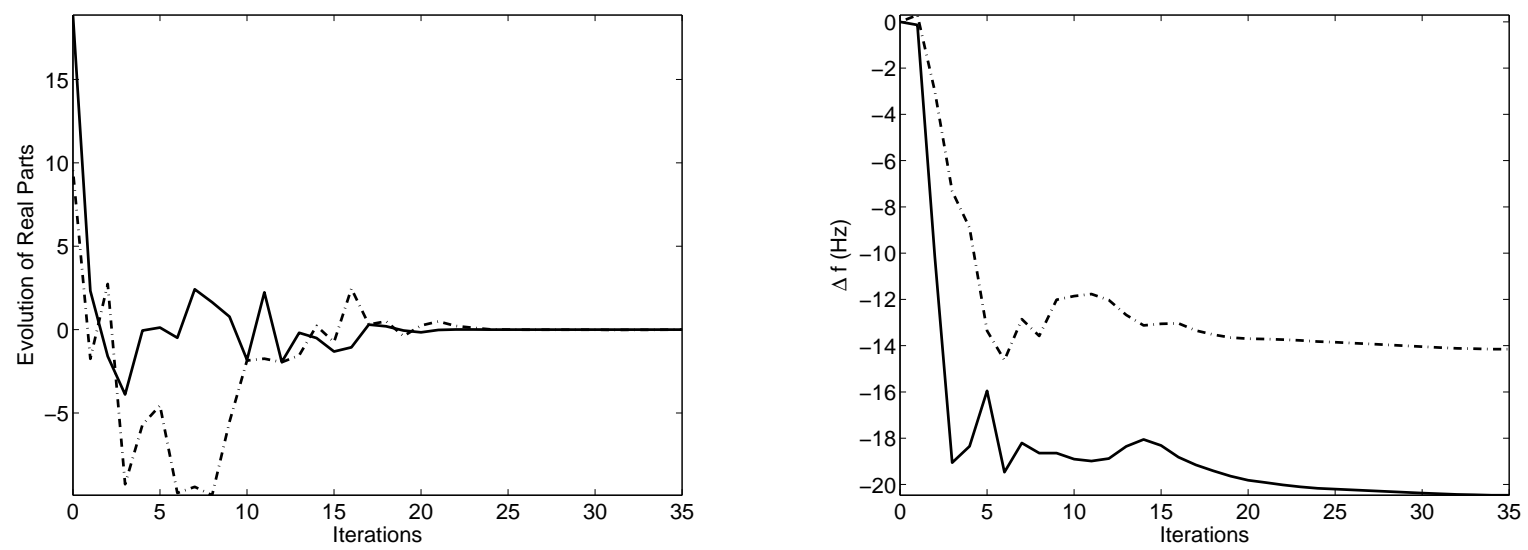

c

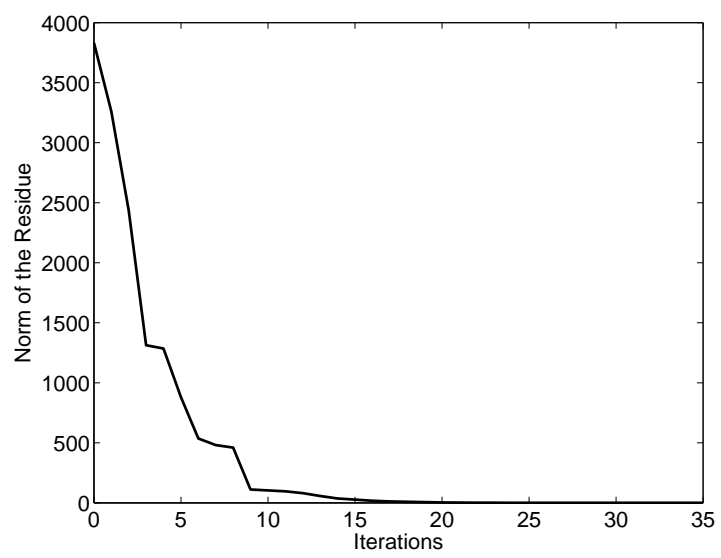

Figure 20: Evolution of parameters with iterations $(\mu=0.29)$. (a) Real Parts: $-1^{\text {st }}$ mode, -.- $2^{\text {nd }}$ mode; (b) Frequencies: $-1^{\text {st }}$ mode, -.- $2^{\text {nd }}$ mode; (c) Norm of Residue 